\title{
Structure and Orientation of a G Protein Fragment in the Receptor Bound State from Residual Dipolar Couplings
}

\author{
Bernd W. Koenig ${ }^{1,2 *}$, Georg Kontaxis ${ }^{2}$, Drake C. Mitchell ${ }^{3}$ \\ John M. Louis ${ }^{2}$, Burton J. Litman ${ }^{3}$ and Ad Bax ${ }^{2 *}$
}

${ }^{1}$ Structural Biology Institute IBI-2, Research Center Jülich D-52425 Jülich, Germany

${ }^{2}$ Laboratory of Chemical Physics, NIDDK, National Institutes of Health, Bethesda MD 20892-0520, USA

${ }^{3}$ Laboratory of Membrane Biophysics and Biochemistry NIAAA, National Institutes of Health, Rockville, MD 20852 USA
Residual dipolar couplings for a ligand that is in fast exchange between a free state and a state where it is bound to a macroscopically ordered membrane protein carry precise information on the structure and orientation of the bound ligand. The couplings originate in the bound state but can be detected on the free ligand using standard high resolution NMR. This approach is used to study an analog of the C-terminal undecapeptide of the $\alpha$-subunit of the heterotrimeric $G$ protein transducin when bound to photo-activated rhodopsin. Rhodopsin is the major constituent of disk-shaped membrane vesicles from rod outer segments of bovine retinas, which align spontaneously in the NMR magnet. Photo-activation of rhodopsin triggers transient binding of the peptide, resulting in measurable dipolar contributions to ${ }^{1} J_{\mathrm{NH}}$ and ${ }^{1} J_{\mathrm{CH}}$ splittings. These dipolar couplings report on the time-averaged orientation of bond vectors in the bound peptide relative to the magnetic field, i.e. relative to the membrane normal. Approximate distance restraints of the bound conformation were derived from transferred NOEs, as measured from the difference of NOESY spectra recorded prior to and after photo-activation. The N-terminal eight residues of the bound undecapeptide adopt a near-ideal $\alpha$-helical conformation. The helix is terminated by an $\alpha_{\mathrm{L}}$ type C-cap, with Gly9 at the $C^{\prime}$ position in the center of the reverse turn. The angle between the helix axis and the membrane normal is $40^{\circ}( \pm 4)^{\circ}$. Peptide protons that make close contact with the receptor are identified by analysis of the NOESY cross-relaxation pattern and include the hydrophobic $\mathrm{C}$ terminus of the peptide.

(C) 2002 Elsevier Science Ltd. All rights reserved

Keywords: NMR; membrane protein; rhodopsin; transferred dipolar couplings; transducin chemokines. More than $50 \%$ of all pharmaceutical drugs target GPCRs. Specific binding of drug molecules modifies the activation state of the receptor and structural aspects of selective recognition between $G$ proteins and their receptors play a key role in understanding intercellular signaling. ${ }^{1,2}$ However, efforts to determine the high resolution structure of an intact functionally active complex between a G protein and its GPCR so far have remained unsuccessful. Instead, models of such complexes have been proposed that rely on structural studies of individual components and analysis of sites and modes of interaction. ${ }^{2-5}$

In the retina, binding of the GDP-bound form of the heterotrimeric $G$ protein transducin $\left(G_{t}\right)$ to the light activated MII intermediate of the 
photoreceptor rhodopsin initiates the visual signal transduction cascade. X-ray structures are now available for dark-adapted rhodopsin ${ }^{6}$ and GDP-bound transducin. ${ }^{7}$ Complementary structural information on bovine rhodopsin comes from $\mathrm{EPR},{ }^{8,9}$ electron diffraction, ${ }^{10,11}$ high resolution NMR, ${ }^{12,13}$ and solid state NMR. ${ }^{14,15}$ Several regions of transducin that are involved in binding to MII have been identified. ${ }^{16,17,4}$ These include the $C$ terminus of the $\alpha$-subunit of transducin $\left(G_{t} \alpha(340-350)\right)$, for which peptide analogs compete with $G_{t}$ for binding to rhodopsin and stabilize its active MII conformation. . $^{1,18}$

The structure of the MII-bound $G_{t} \alpha(340-350)$ peptide previously has been studied by high resolution NMR exploiting transferred NOEs, but yielded partly contradictory results. ${ }^{19,20}$ Here, we demonstrate that measurement of residual dipolar couplings in an analog of $G_{t} \alpha(340-350)$, bound to MII, complements transferred NOE data and results in a more detailed view of the structure of the bound ligand peptide. Equally importantly, dipolar couplings also yield the orientation of the peptide relative to the membrane normal, thereby facilitating assembly of the molecular pieces of the puzzle.

Structures of tight complexes between proteins and their ligands are amenable to multi-dimensional high resolution NMR, provided the rotational correlation time of such complexes remains sufficiently short. In practice this puts an NMR limit of ca $30-50 \mathrm{kDa}$ on the size of such complexes. ${ }^{21}$ However, in combination with suitable isotope labeling schemes, systems above this limit are also accessible. ${ }^{22-24}$

The size limitations for tight complexes do not apply to the NMR study of soluble ligands that transiently bind to macromolecules. Provided the exchange rate between the free and bound forms of the ligand is comparable to or faster than the rate at which cross-relaxation in the bound state takes place, regular solution NMR experiments on the excess of free ligand can be used to study the conformation of the bound ligand. The very efficient cross-relaxation processes in the slowly tumbling complex dominate the magnetization transfer processes observed for the rapid equilibrium between free and bound ligands; the cross-relaxation occurring in the free ligand is often negligibly slow. Therefore, NOESY spectra on a dynamically exchanging system containing an excess of free ligand, in rapid exchange with bound ligand, can be used to detect spatial proximity of protons in the bound form. ${ }^{25-27}$ However, interpretation of the resulting transferred $\mathrm{NOE}$ (TrNOE) data may be challenging due to the possibility of indirect magnetization transfer effects and intermolecular cross-relaxation between ligand and partner. ${ }^{28}$ When binding to a large, fully oriented particle, interproton magnetization transfer is governed by the rules of solid state nuclear spin exchange and not by relaxation theory. ${ }^{29}$ For example, if a pair of protons in the bound state is oriented at an angle close to $54.7^{\circ}$ with respect to the magnetic field, their dipolar interaction is very small and the rate of magnetization exchange is very low. The case where $G_{t} \alpha(340-350)$ binds to rhodopsin, anchored in the disk membranes isolated from retinal rod outer segments, is intermediate between the solid state case and the situation normally encountered in transferred NOE studies of ligand binding to soluble proteins. Therefore, it is not clear a priori how reliable the distance restraints are that can be extracted from a TrNOE spectrum for the $G_{t} \alpha(340-350)$ analog.

Here, we complement the TrNOE method with the measurement of residual dipolar couplings. Residual dipolar couplings report on the timeaveraged orientation of internuclear bond vectors with respect to the magnetic field, thereby providing important global restraints for structure refinement. ${ }^{30}$ Experimentally measured residual one-bond dipolar couplings are directly related to the ensemble average over $\left(3 \cos ^{2} \theta-1\right) / 2$, where $\theta$ is the angle between the bond vector and the magnetic field, independent of the time scale. They therefore are not subject to the above mentioned problem encountered in interpreting TrNOE data. Dipolar couplings have proven to be particularly useful in defining relative alignment of individual units in multidomain proteins and macromolecular complexes, even in the absence of long range NOEs between them. ${ }^{31-33}$ In addition, they have been shown to improve the local geometry of protein structures. ${ }^{30}$ Measurement of residual dipolar couplings requires partial alignment of the solute with respect to the magnetic field. A small degree of net alignment can be imparted onto solute molecules by placing them in an environment that is not spherically symmetric. For example, steric interactions with a dilute liquid crystalline phase of oriented phospholipid bilayers can be used for this purpose, ${ }^{34}$ or weak electrostatic interactions in a medium containing one to two weight percent of charged, rod-shaped oriented virus particles ${ }^{35-37}$ can induce the required weak alignment. Transient, nonspecific binding to the highly charged surface of oriented purple membrane fragments can also be used for inducing a non-zero average over $\left(3 \cos ^{2} \theta-1\right) / 2 .{ }^{38,39}$

For unbound, freely diffusing peptides, there is no net orientation relative to the magnetic field and for each bond vector in the peptide the time average over $\left(3 \cos ^{2} \theta-1\right) / 2$ equals zero. Intact individual rhodopsin-rich rod outer segments of bovine retinas align spontaneously in high field NMR magnets, with the membrane normal parallel with the magnetic field. ${ }^{40,41}$ We have recently shown that individual disk membranes, isolated from the retinal rod outer segments by the Ficoll flotation method ${ }^{42}$ also align with their membrane normal parallel with the magnetic field. ${ }^{43}$ Transient, specific binding of a peptide analog of $\mathrm{G}_{\mathrm{t}} \alpha(340-350)$ to the MII photointermediate of such oriented rhodopsin molecules results in non-zero 


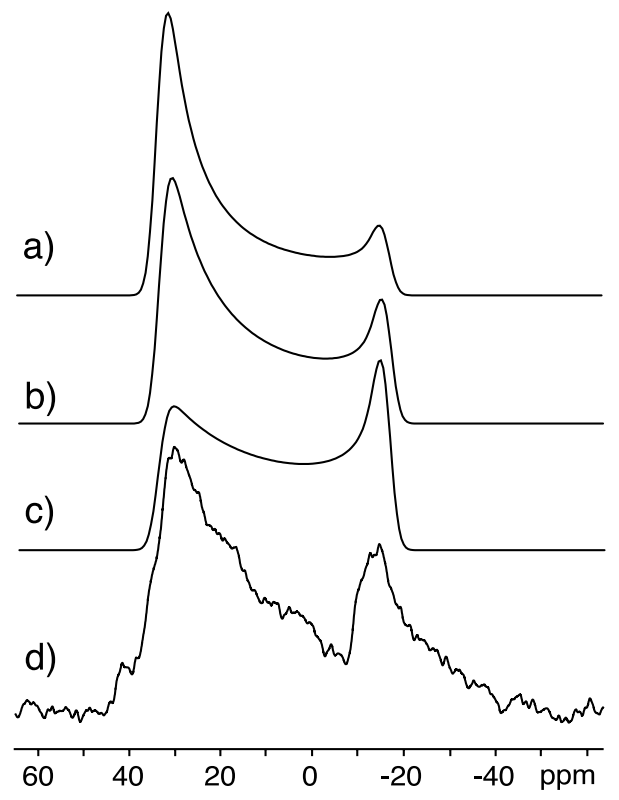

Figure 1. ${ }^{31} \mathrm{P}$ NMR spectra of disk membrane phospholipids. (a)-(c) Spectra simulated for phosphoplipids embedded in the surface of an oblate spheroid with its unique axis parallel with the magnetic field and an aspect ratio of (a) 2.5:1, (b) 2:1, and (c) 1.5:1. Spectra are simulated under the assumption of fast axial rotation of the phospholipid in the bilayer, resulting in an axially symmetric CSA tensor, but no translational diffusion. Simulated spectra assume a single axially symmetric CSA value, $v_{\|}-v_{\perp}=49 \mathrm{ppm}$, for the three major lipid constituents of the membrane. (d) Experimental spectrum of rhodopsin-containing disk membranes.

values for $\left(3 \cos ^{2} \theta-1\right) / 2{ }^{43}$ Here, we extend this measurement to the uniformly ${ }^{13} \mathrm{C} /{ }^{15} \mathrm{~N}$-labeled peptide and demonstrate application of these transferred residual dipolar couplings (TrDCs) to structure determination of the bound peptide. Combined use of TrDCs and TrNOEs yields a high quality structure of the bound peptide and defines the orientation of the MII-bound peptide relative to the membrane normal. Intermolecular relaxation can also be observed and points to the peptide contact surface with rhodopsin. Furthermore, simultaneous structure refinement against fundamentally different experimental parameters reduces the risk that potential artifacts of TrNOEderived distance restraints, resulting from spin diffusion or intermolecular cross-relaxation, or from errors in TrDCs caused by differential mobility of bond vectors, get manifested in a biased structure.

\section{Results}

We previously have demonstrated the feasibility of measuring residual dipolar couplings for a peptide mimicking the C-terminal fragment of transducin. ${ }^{43}$ Here, we resort to uniform ${ }^{13} \mathrm{C}$ and ${ }^{15} \mathrm{~N}$ enrichment of the peptide, in order to measure as large as possible a set of one-bond heteronuclear dipolar couplings. TrDC and TrNOE measurements of the type utilized here, when transiently binding to a very large particle, require high offrates, i.e. weak binding constants. The S2 peptide corresponds to $G_{t} \alpha(340-350)-K 341 R-C 347 S$, and was chosen here for its lower affinity to MII, resulting in an enhanced off-rate. MII stabilization by the S2 peptide was confirmed by spectrophotometry. ${ }^{43}$ An additional benefit of the C347S substitution is the elimination of the need to add a strong reducing agent to the sample, necessary for the native peptide to avoid disulfide formation. The NOE patterns we observe in this region of the peptide are quite similar to those reported by Kisselev et al., ${ }^{20}$ indicating that the C347S substitution does not cause any major structural rearrangement.

After photo-activation, rhodopsin exists as an equilibrium of MI and MII states, whereas MII itself exists in two isochromic substates, MIIa and MIIb. ${ }^{44}$ The MI to MII conversion is highly endothermic $\left(160 \mathrm{~kJ} \mathrm{~mol}^{-1}\right) .{ }^{44}$ In order to minimize population of the MI state, measurements were carried out at higher temperatures $\left(10{ }^{\circ} \mathrm{C}\right.$ and $20^{\circ} \mathrm{C}$ ) than used in previous NMR studies. MIIa and MIIb interconvert rapidly and are associated with distinct steps in deprotonation of the Schiff base and proton uptake. ${ }^{45}$

\section{Alignment of rhodopsin-rich disks}

Measurement of dipolar couplings in the peptide, which transiently binds to rhodopsin anchored in the oriented surface of disk membranes prepared from retinal rod outer segments requires a significant degree of net orientation of these disks. However, at the high concentration needed for NMR studies, the alignment of these rhodopsin-loaded flattened unilamellar vesicles is a compromise between minimizing their aggregation, which requires low ionic strength, and minimizing their osmotic swelling, which requires high ionic strength of the surrounding solvent. Empirically, a compromise is found that contains $5.8 \mathrm{mg}$ disks per $\mathrm{ml}(63 \mu \mathrm{M}$ rhodopsin) in $10 \mathrm{mM}$ Hepes buffer, and $20 \mathrm{mM} \mathrm{KCl}$. Under these conditions, the ${ }^{31} \mathrm{P}$ NMR spectrum of the phospholipids contained in the disk membranes corresponds to that of a distribution on an oblate spheroid with an aspect ratio of ca 2 (Figure 1), and the unique (short) axis parallel with the magnetic field. This small aspect ratio indicates that the vesicles are swollen considerably, which results in a distribution of alignment orientations of bound peptide relative to the direction of the magnetic field. The effective alignment of bound peptide is a weighted average over the surface of the ellipsoid and is scaled down by a factor of 0.4 relative to the alignment expected for binding to a perfectly flat disk. Nevertheless, these conditions permit measurement of significant dipolar couplings in the S2 peptide. It is important to note that 


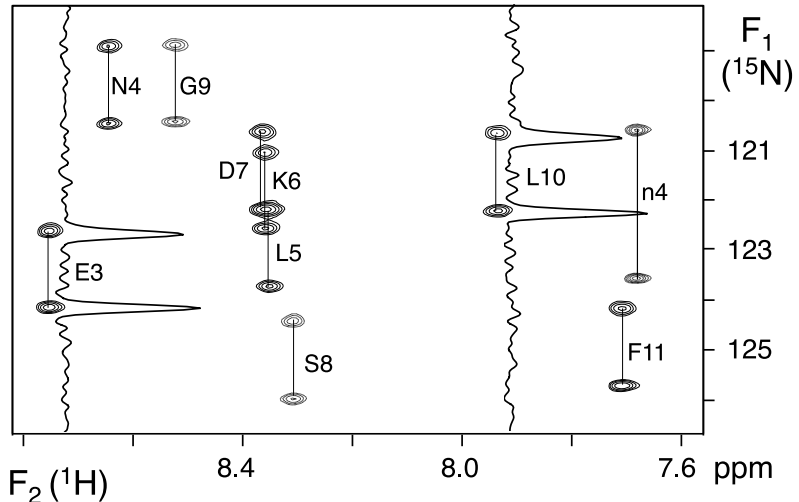

Figure 2. Section of the ${ }^{1} \mathrm{H}-{ }^{15} \mathrm{~N}$ HSQC spectrum, recorded in the ${ }^{1} \mathrm{H}$-coupled mode, at $10{ }^{\circ} \mathrm{C}$ immediately following photo-activation. The two inserted $F_{1}$ crosssections highlight the relaxation interference effect. Due to faster exchange, the relaxation interference effect is more pronounced at $20^{\circ} \mathrm{C}$. Correlations for Gly9, Ser8, and the Asn4 side-chain amide protons (labeled n4) are folded as a result of the small spectral width in the indirect dimension.

the precise value of the aspect ratio has no impact on the use of the dipolar coupling because the alignment strength used in structure calculations is a fitted parameter, which is a complex function of many factors. These include, besides the aspect ratio of the disks, the binding kinetics, the rotational diffusion and relaxation rates in the bound state, and the degree of internal dynamics in the bound state.

\section{Transient peptide binding to a large particle}

Exposure of the dark-adapted rhodopsin to bright light triggers transient binding of the S2 peptide to rhodopsin. At our experimental conditions (2.6 $\mathrm{mM}$ S2 peptide, $63 \mu \mathrm{M}$ rhodopsin, $20^{\circ} \mathrm{C}$ ) this causes the transverse relaxation rate of peptide amide ${ }^{15} \mathrm{~N}$ spins to increase from $6.6 \mathrm{~s}^{-1}$ to about $28 \mathrm{~s}^{-1}$. The resulting line broadening is considerable and gives rise to a pronounced doublet asymmetry in the proton-coupled ${ }^{15} \mathrm{~N}-{ }^{1} \mathrm{H}$ HSQC spectrum, caused by relaxation interference between the ${ }^{15} \mathrm{~N}$ chemical shift anisotropy (CSA) and the one-bond ${ }^{15} \mathrm{~N}-{ }^{1} \mathrm{H}$ dipolar interaction. ${ }^{46}$ This effect is particularly strong at $20^{\circ} \mathrm{C}^{43}$ but as discussed below, it becomes less pronounced at lower temperature where both the on-and off-rates are lower and the MI-MII equilibrium is more shifted towards MI (Figure 2).

The rhodopsin-rich rod outer segment disk has a diameter of several microns and a mass of about 50 gigadalton. For NMR purposes it can be considered as a solid, non-tumbling object, but with relatively fast axial rotational diffusion of rhodopsin within the membrane, with a rotational correlation time of ca $20 \mu \mathrm{s}{ }^{47}$ Importantly, this rotational diffusion is faster than the inverse of the static homonuclear and heteronuclear dipolar interactions. Therefore, upon binding to rhodopsin, rotational diffusion of the S2 peptide about an axis that is parallel with the local bilayer normal reduces all dipolar couplings by a factor $\left(3 \cos ^{2} \alpha-1\right) / 2$, where $\alpha$ is the angle between the local bilayer normal and the magnetic field. The decay rate, $\rho$, of transverse S2 magnetization in the bound state depends on where on the vesicle surface the S2 peptide binds. Decay of transverse magnetization for the $\mathrm{S} 2$ peptide bound in a region where $\alpha$ is small or close to $90^{\circ}$ will be very fast, essentially occurring at the rate that is observed in the solid state and which is determined by the inverse of the static ${ }^{1} \mathrm{H}-{ }^{1} \mathrm{H}$ dipolar interactions (i.e. on a time scale of ca $50 \mu \mathrm{s}$ ). For bound times longer than $50 \mu \mathrm{s}$, an increasing fraction of transverse S2 peptide magnetization will decay prior to release of the peptide into the solvent, thereby contributing less to the observed dipolar splittings and to the above mentioned relaxation-interference-induced asymmetry of the ${ }^{1} \mathrm{H}-\left\{{ }^{15} \mathrm{~N}\right\}$ doublet.

Quantitatively, the TrDCs can be calculated by considering the system as a two-site exchange problem, where in one site (free in solution) its relaxation is negligibly slow and the dipolar coupling is zero, whereas in the second site (bound state) the transverse relaxation rate, $\rho$, is fast and the dipolar coupling equals $D_{\mathrm{b}}$ (in units of radian $\mathrm{s}^{-1}$ ). The average life time of a free ligand is defined as $\tau_{\mathrm{f}}\left(\tau_{\mathrm{f}}=1 / k_{\mathrm{on}}[\mathrm{P}]\right.$; with $k_{\mathrm{on}}$ the on-rate and $[\mathrm{P}]$ the concentration of unligated MII) and the bound time, $\tau_{\mathrm{b}}=k_{\mathrm{off}}^{-1}$. In the applicable limit where $\left(\tau_{\mathrm{f}} \tau_{\mathrm{b}}\right)^{-1} \ll\left(\rho-\tau_{\mathrm{f}}^{-1}+\tau_{\mathrm{b}}^{-1}\right)^{2}$, one obtains for the observed dipolar splitting of the free component, $D_{\text {obs }}:{ }^{48}$

$$
D_{\mathrm{obs}}=\left(\tau_{\mathrm{f}} \tau_{\mathrm{b}}\right)^{-1} D_{\mathrm{b}} /\left[\left(\rho-\tau_{\mathrm{f}}^{-1}+\tau_{\mathrm{b}}^{-1}\right)^{2}+D_{\mathrm{b}}^{2}\right]
$$

In this limit, the line width, $\Delta v_{1 / 2}$, of the free component is given by:

$$
\begin{aligned}
& \Delta \nu_{1 / 2}=\pi^{-1}\left\{\tau_{\mathrm{f}}^{-1}-\left(\tau_{\mathrm{f}} \tau_{\mathrm{b}}\right)^{-1}\left(\rho-\tau_{\mathrm{f}}^{-1}+\tau_{\mathrm{b}}^{-1}\right) /\right. \\
& \left.\times\left[\left(\rho-\tau_{\mathrm{f}}^{-1}+\tau_{\mathrm{b}}^{-1}\right)^{2}+D_{\mathrm{b}}^{2}\right]\right\}
\end{aligned}
$$

In equations (1), $D_{\mathrm{b}}$ and $\rho$ are functions of $\alpha$, and the full signal is obtained by integrating equations (1) over the surface of the vesicle. Equation (1a) highlights that linear scaling between the observed TrDC coupling $\left(D_{\text {obs }}\right)$ and the dipolar coupling in the bound state $\left(D_{\mathrm{b}}\right)$ requires that the off-rate, $k_{\text {off }}$ is much larger than the dipolar coupling in the bound state.

Observed residual dipolar couplings are proportional to $\left(3 \cos ^{2} \theta-1\right) / 2$, where $\theta$ is the angle between an internuclear vector and the local bilayer normal which determines the value of $D_{\mathrm{b}}$, but are also a function of $\rho$. Under the simplifying assumptions that $\rho$ is independent of $\theta$, and $D_{\mathrm{b}} \ll$ $\left(\rho-\tau_{f}^{-1}+\tau_{b}^{-1}\right)$, the averaging over the surface of the oblate-shaped rod outer segment vesicle simply results in a uniform scaling factor of the dipolar couplings. So, observed residual dipolar couplings are directly proportional to 

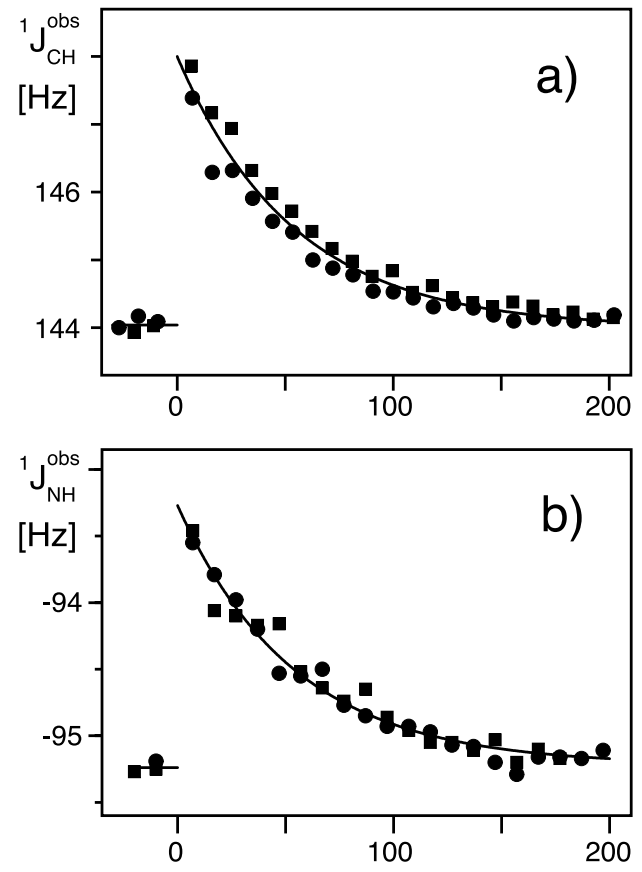

Time after Rhodopsin Activation [min]

Figure 3. Time dependence of ${ }^{15} \mathrm{~N}-{ }^{1} \mathrm{H}$ and ${ }^{13} \mathrm{C}^{\alpha}-{ }^{1} \mathrm{H}^{\alpha}$ one-bond $J+D$ splittings of (a) Asp7 $\mathrm{C}^{\alpha}-\mathrm{H}^{\alpha}$ and (b) Gly $9 \mathrm{~N}-\mathrm{H}^{\mathrm{N}}$ in the $\mathrm{S} 2$ peptide, at $10{ }^{\circ} \mathrm{C}$, observed after photo-activation of rhodopsin at $t=0$. The time after photo-activation refers to the mid-point of the NMR measurement. Experiments were conducted twice, and data points from the separate series of experiments are represented by filled squares and circles, respectively.

$\left(3 \cos ^{2} \theta-1\right) / 2$. For a unique bound conformation of the peptide, the net alignment tensor for the peptide therefore will be axially symmetric.

${ }^{1} \mathrm{H}-{ }^{1} \mathrm{H}$ longitudinal spin exchange in the bound state, which forms the basis of the transferred NOE effect, also depends on $\alpha$. In contrast to the case of transverse magnetization, longitudinal spin exchange does not lead to the same loss of signal, but redistributes the magnetization of a given proton over its neighbors with which it has a large ${ }^{1} \mathrm{H}-{ }^{1} \mathrm{H}$ dipolar interaction. This kind of magnetization transfer is not the truly stochastic process which underlies the NOE, but is governed by deterministic spin exchange, defined by the static dipolar couplings. ${ }^{29}$ In contrast, for peptides bound near $\alpha=54.7^{\circ}$ (the so-called magic angle) rotational diffusion of rhodopsin about the bilayer normal strongly reduces both the dipolar broadening and the effective dipolar coupling. In this region, ${ }^{1} \mathrm{H}-{ }^{1} \mathrm{H}$ magnetization transfer again becomes a stochastic NOE-type process, which generally takes place at a much slower rate than applies in the static case. In practice, the distribution of peptide orientations in the bound state gives rise to a complex behavior of the observed magnetization buildup in the transferred NOE experiment and therefore requires careful analysis to exclude the effects of indirect magnetization transfer processes (spin diffusion).

\section{Time dependence of dipolar couplings}

HSQC spectra correlating directly bonded ${ }^{1} \mathrm{H}-{ }^{15} \mathrm{~N}$ or ${ }^{1} \mathrm{H}-{ }^{13} \mathrm{C}$ spin pairs were recorded without ${ }^{1} \mathrm{H}$ decoupling in the $F_{1}$ dimension (Figure 2). The splitting of the $F_{1}$ doublets for amide and methine sites corresponds to the sum of the onebond ${ }^{1} J$ coupling and the residual dipolar coupling, ${ }^{1} D$, of the two interacting spins. For methylene sites the center line of the regular ${ }^{13} \mathrm{C}-\left\{\mathrm{H}_{2}\right\}$ triplet is invisible and the observed splitting corresponds to the sum of the two individual ${ }^{1} J_{\mathrm{CH}}+{ }^{1} D_{\mathrm{CH}}$ couplings. A quartet is observed for rapidly rotating methyl groups and the dipolar contribution to the splitting of the outer lines can be used directly to constrain the orientation of the $\mathrm{C}-\mathrm{CH}_{3}$ bond axis. $^{49}$

At a $2.6 \mathrm{mM}$ concentration of uniformly ${ }^{15} \mathrm{~N} /$ ${ }^{13} \mathrm{C}$-labeled peptide, acquisition of high sensitivity $2 \mathrm{D}{ }^{1} \mathrm{H}-{ }^{15} \mathrm{~N}$ and ${ }^{1} \mathrm{H}-{ }^{13} \mathrm{C}$ correlation spectra requires only two scans per $t_{1}$-increment, allowing the recording of a complete HSQC spectrum in less than ten minutes. Such rapid data accumulation is necessary for monitoring the change in dipolar coupling during the relatively fast decay of the photo-activated MII state of rhodopsin. Spectral overlap in the ${ }^{1} \mathrm{H}-{ }^{13} \mathrm{C}$ HSQC spectrum prohibits accurate dipolar coupling measurement for several of the aliphatic resonances. Recording of multiple 3D spectra, in which such resonance overlap would be completely absent, proved to be impractical due to the limited lifetime of the MII state.

Several series of ${ }^{1} \mathrm{H}$-coupled ${ }^{1} \mathrm{H}-{ }^{15} \mathrm{~N}$ and ${ }^{1} \mathrm{H}-{ }^{13} \mathrm{C}$ HSQC experiments were recorded prior to and after light activation, each time using a fresh rhodopsin preparation. The experimentally derived dipolar couplings and TrNOEs represent the difference relative to the dark-adapted state. Therefore, effects of interactions with rhodopsin or membrane sites, which are not affected by the photoactivation, are subtracted out and the extracted dipolar couplings therefore reflect the MII-bound state.

One-bond ${ }^{15} \mathrm{~N}-{ }^{1} \mathrm{H}$ and ${ }^{13} \mathrm{C}-{ }^{1} \mathrm{H}$ splittings each change by specific amounts after photo-activation, but return to their dark-adapted value in an exponential fashion in subsequent spectra. The magnitude and time course of this change are highly reproducible (Figure 3 ). The time course of splittings observed after light activation, $J^{\text {obs }}(t)$, is described by:

$$
{ }^{1} J^{\mathrm{obs}}(t)={ }^{1} J^{\mathrm{dark}}+{ }^{1} D \exp (-t / \tau)
$$

where ${ }^{1} J^{\text {dark }}$ is the coupling in the dark-adapted state, ${ }^{1} D$ is the residual dipolar coupling at $t=0$, and the time constant $\tau$ is the exponential decay constant of the peptide-binding MII state of rhodopsin. The exponential decay curves of all couplings measured after bleaching at a given temperature were fitted simultaneously to optimize the selection of a single $\tau$ value. Decay constants of 54 minutes at $10^{\circ} \mathrm{C}$ and 15 minutes at $20^{\circ} \mathrm{C}$ were obtained. On the basis of the 


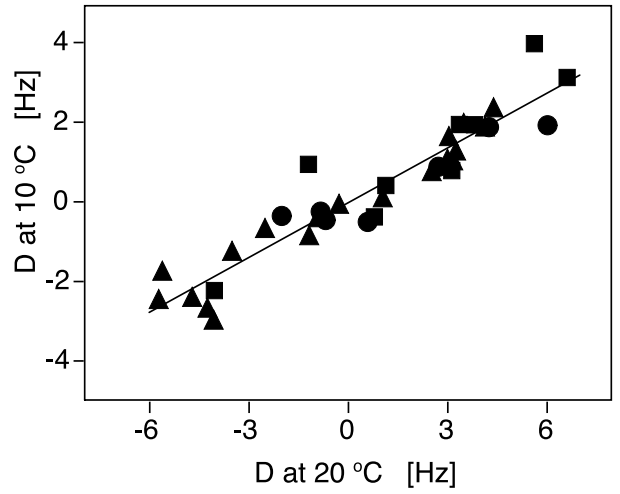

Figure 4. Correlation between ${ }^{15} \mathrm{~N}-{ }^{1} \mathrm{H}(\bullet),{ }^{13} \mathrm{C}^{\alpha}-{ }^{1} \mathrm{H}^{\alpha}$ (ם), and side-chain ${ }^{13} \mathrm{C}-{ }^{1} \mathrm{H}(\boldsymbol{\Delta})$ dipolar couplings in the S2 peptide measured at $10{ }^{\circ} \mathrm{C}$ and at $20^{\circ} \mathrm{C}$, immediately after photo-activation of rhodopsin.

exponential fit, each coupling was extrapolated to $t=0$, i.e. to the midpoint of bleaching. The difference between the extrapolated coupling at $t=0$ and the coupling measured from the dark-adapted sample represents the residual dipolar coupling.

Dipolar couplings measured at $10{ }^{\circ} \mathrm{C}$ and $20^{\circ} \mathrm{C}$ have different magnitudes resulting from changes in the binding kinetics (equations (1)) and population of the peptide-binding MII state. However, assuming structure and orientation of the peptide relative to the normal of the disk are identical at the two temperatures, and that in both cases the off-rate is much larger than the dipolar coupling in the bound state, the ratio of values measured at $10^{\circ} \mathrm{C}$ and $20^{\circ} \mathrm{C}$ should be the same for all dipolar couplings. Indeed, a good correlation with a linear correlation coefficient of $R=0.94$ and a slope of 2.06 is found for the couplings measured at $10{ }^{\circ} \mathrm{C}$ and $20^{\circ} \mathrm{C}$ (Figure 4). Assuming the relative error is the same at both temperatures, the data recorded at $20{ }^{\circ} \mathrm{C}$ may be scaled down by a factor of 2.06 and subsequently averaged with the $10{ }^{\circ} \mathrm{C}$ data. The pairwise rmsd between the $10^{\circ} \mathrm{C}$ and the scaled $20^{\circ} \mathrm{C}$ data equals $0.56 \mathrm{~Hz}$ and $0.76 \mathrm{~Hz}$ for the backbone ${ }^{15} \mathrm{~N}-{ }^{1} \mathrm{H}$ and ${ }^{13} \mathrm{C}^{\alpha}-{ }^{1} \mathrm{H}^{\alpha}$ couplings, respectively, and $0.46 \mathrm{~Hz}$ for the side-chain ${ }^{13} \mathrm{C}^{1}-{ }^{1} \mathrm{H}$ interactions. The pairwise rmsd equals two times the error in the individual couplings, and averaging the $10{ }^{\circ} \mathrm{C}$ and scaled $20^{\circ} \mathrm{C}$ data therefore yields random measurement uncertainties of $0.28 \mathrm{~Hz}, \quad 0.38 \mathrm{~Hz}$, and $0.23 \mathrm{~Hz}$ for ${ }^{15} \mathrm{~N}-{ }^{1} \mathrm{H}$, ${ }^{13} \mathrm{C}^{\alpha}-{ }^{1} \mathrm{H}^{\alpha}$, and side-chain couplings, respectively.

A total of nine backbone ${ }^{15} \mathrm{~N}-{ }^{1} \mathrm{H}$ residual dipolar couplings were measured for the 11-residue S2 peptide. Amide protons of Ile1 and Arg2 could not be observed due to fast exchange with solvent protons. Although the upfield ${ }^{15} \mathrm{~N}-\left\{{ }^{1} \mathrm{H}\right\}$ of Leu5 overlaps the downfield ${ }^{15} \mathrm{~N}-\left\{{ }^{1} \mathrm{H}\right\}$ component of Asp7 at $10^{\circ} \mathrm{C}$, both couplings are resolved at $20{ }^{\circ} \mathrm{C}$. Overlap of the ${ }^{13} \mathrm{C}^{\alpha}-{ }^{1} \mathrm{H}^{\alpha}$ correlations of Arg2 and Leu5 prohibited measurement of their ${ }^{1} D_{\mathrm{C} \alpha \mathrm{H} \alpha}$ dipolar couplings at both temperatures, but couplings for the nine remaining ${ }^{13} \mathrm{C}^{\alpha}-{ }^{1} \mathrm{H}^{\alpha}$ pairs
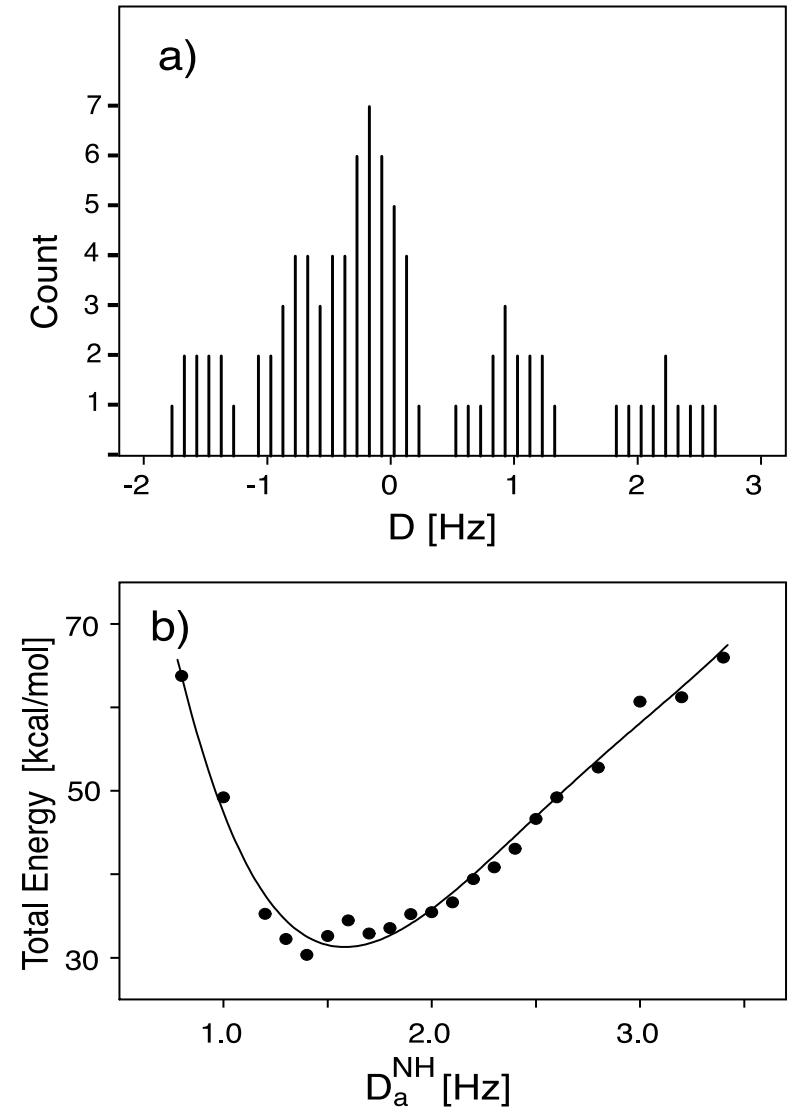

Figure 5. Determination of the magnitude, $D_{\mathrm{a}}^{\mathrm{NH}}$, of the molecular alignment tensor of the S2 peptide after photo-activation of rhodopsin at $10^{\circ} \mathrm{C}$. (a) Histogram representation of ${ }^{15} \mathrm{~N}-{ }^{1} \mathrm{H}$ and ${ }^{13} \mathrm{C}^{\alpha}-{ }^{1} \mathrm{H}^{\alpha}$ residual dipolar couplings. Couplings are normalized relative to the ${ }^{15} \mathrm{~N}-{ }^{1} \mathrm{H}$ values, i.e. ${ }^{13} \mathrm{C}^{\alpha}-{ }^{1} \mathrm{H}^{\alpha}$ couplings are scaled by -0.48 to account for the different gyromagnetic ratio of ${ }^{13} \mathrm{C}$ versus ${ }^{15} \mathrm{~N}$ and the difference in bond length. ${ }^{53}$ Five neighboring bins, centered at the measured coupling and $0.1 \mathrm{~Hz}$ wide each, are incremented for each observed dipolar coupling in order to account for experimental uncertainty. (b) Total energy of 20 calculated lowest energy structures as a function of the $D_{\mathrm{a}}^{\mathrm{NH}}$ value used in the structure calculation. This latter procedure tends to underestimate $D_{\mathrm{a}}^{\mathrm{NH}}$ and final calculations were carried out using the highest $D_{\mathrm{a}}^{\mathrm{NH}}$ value still yielding low energy structures $\left(D_{\mathrm{a}}^{\mathrm{NH}}=1.7 \mathrm{~Hz}\right)$. The plotted total energy represents the average over the 20 lowest energy structures (out of 100 calculated).

and an additional 20 side-chain ${ }^{13} \mathrm{C}-{ }^{1} \mathrm{H}$ couplings were measured.

\section{Magnitude of molecular alignment tensor}

As mentioned above, for a unique bound conformation the molecular alignment tensor must be axially symmetric, and the dipolar coupling between nuclei $P$ and $Q$ is then described by:

$$
D_{\mathrm{PQ}}(\theta)=S D_{\mathrm{a}}^{\mathrm{PQ}}\left(3 \cos ^{2} \theta-1\right)
$$

where $S$ is the generalized order parameter ${ }^{50}$ for internal motion of vector $\mathrm{PQ}$, and $D_{\mathrm{a}}^{\mathrm{PQ}}$ is commonly 
referred to as the magnitude of the residual dipolar coupling tensor, given by:

$$
D_{\mathrm{a}}^{\mathrm{PQ}}=-\left(\mu_{0} / 4 \pi\right) \gamma_{\mathrm{P}} \gamma_{\mathrm{Q}} h A_{\mathrm{a}} / 4 \pi^{2} r_{\mathrm{PQ}}^{3}
$$

with $h$ being Planck's constant, $\gamma_{\mathrm{x}}$ the gyromagnetic ratio of nucleus $X, \mu_{0}$ is the magnetic permeability of vacuum, $r_{\mathrm{PQ}}$ the $\mathrm{PQ}$ internuclear distance, $\theta$ the angle between the orientation of $\mathrm{PQ}$ and the unique axis of the molecular alignment tensor $A$, and $A_{\mathrm{a}}$ the axial component of this tensor. $S^{2}$ values of backbone bond vectors in structured proteins typically range from 0.8 to 0.9 , i.e. $S$ is $0.93( \pm 0.03) .{ }^{51}$ Here we assume $\boldsymbol{S}$ to be the same for all backbone $\mathrm{N}-\mathrm{H}$ and $\mathrm{C}^{\alpha}-\mathrm{H}^{\alpha}$ vectors of the bound S2 peptide, which introduces an error that is smaller than the random measurement error in this coupling. This constant $S$ is then absorbed into the value of $D_{a}$, which is determined in the manner described below.

As indicated by equation (3b), the $D_{\mathrm{a}}^{\mathrm{NH}} / D_{\mathrm{a}}^{\mathrm{C} \alpha \mathrm{H} \alpha}$ ratio is a constant determined by $\gamma_{\mathrm{N}}\left\langle r_{\mathrm{N}-\mathrm{H}}^{-3}\right\rangle /$ $\gamma_{\mathrm{C}}\left\langle r_{\mathrm{C}-\mathrm{H}}^{-3}\right\rangle$, where \langle\rangle brackets refer to averaging over the ensemble. Experimentally, a $D_{\mathrm{a}}^{\mathrm{NH}}$ \% $D_{\mathrm{a}}^{\mathrm{C \alpha H \alpha}}=-0.48$ ratio has been found. ${ }^{52}$ The magnitude of $D_{\mathrm{a}}^{\mathrm{NH}}$ depends on the degree of alignment of the peptide and is not known a priori. For large proteins with a random orientational distribution of bond vectors the components of the alignment tensor can be determined from the histogram of measured dipolar couplings. ${ }^{53}$ For an axially symmetric tensor, as applies in the present case, the histogram is expected to resemble a CSA powder pattern spanning the range between $-D_{\mathrm{a}}^{\mathrm{NH}}$ and $+2 D_{\mathrm{a}}^{\mathrm{NH}}$, with high and low intensity shoulders at $-D_{\mathrm{a}}^{\mathrm{NH}}$ and $+2 D_{\mathrm{a}}^{\mathrm{NH}}$, respectively. The histogram of the measured backbone $\mathrm{N}-\mathrm{H}$ and normalized $\mathrm{C}^{\alpha}-\mathrm{H}^{\alpha}$ dipolar couplings of the S2 peptide shows a rather poor resemblance to an axially symmetric powder pattern (Figure 5(a)). This reflects the small size of the peptide, which results in non-uniform sampling of orientational space. However, the observed pattern defines a lower limit: $D_{\mathrm{a}}^{\mathrm{NH}} \geq 1.6 \mathrm{~Hz}$. A final value of $D_{\mathrm{a}}^{\mathrm{NH}}=1.6( \pm 0.2) \mathrm{Hz}$ was determined from conducting a one-dimensional systematic search (Figure 5(b)) in which the total energy of the peptide structure, derived from both TrNOE and residual dipolar coupling data, is plotted as a function of the $D_{\mathrm{a}}^{\mathrm{NH}}$ value used. ${ }^{54}$ Simulations carried out on structures containing small errors in bond vector orientations ${ }^{55}$ indicate that a systematic search as carried out above tends to underestimate the true magnitude of $D_{\mathrm{a}}$ and all final structure calculations were therefore carried out assuming $D_{\mathrm{a}}^{\mathrm{NH}}=1.7 \mathrm{~Hz}$. The positive sign of $D_{\mathrm{a}}^{\mathrm{NH}}$ (taking into account the negative value of $\gamma_{N}$ ) confirms that the unique axis is aligned parallel with the magnetic field, as expected for oblate-shaped rhodopsin-bearing disk membranes, oriented with their short axis parallel with the magnetic field.

\section{TrNOESY difference spectra}

${ }^{1} \mathrm{H}$ and ${ }^{15} \mathrm{~N}$ spectra of the $\mathrm{S} 2$ peptide were assigned using regular ${ }^{15} \mathrm{~N}$-separated TOCSY and NOESY spectra ${ }^{56}$ at $10^{\circ} \mathrm{C}$ for a free peptide sample. ${ }^{1} \mathrm{H}$ chemical shifts are found to be in very close agreement with those reported by Kisselev for the homologous peptide. ${ }^{20}{ }^{13} \mathrm{C}$ shifts were assigned by correlating them with the assigned proton chemical shifts. All chemical shifts have been deposited in the BMRB database (accession number 5376). The spectra of the free S2 peptide remain essentially unperturbed upon addition of dark-adapted rhodopsin, but show considerable line broadening upon light-induced conversion of rhodopsin to the MII state. As mentioned above, no changes in resonance frequencies are observed upon photo-activation.

The NOESY spectrum of the free peptide at $10{ }^{\circ} \mathrm{C}$ shows significant cross-peaks that are of the same sign as the diagonal peaks, indicating that the peptide is in the slow tumbling limit $\left(\omega_{\mathrm{H}} \tau_{\mathrm{c}}>1.1\right)$. These free peptide cross-peaks superimpose on the NOE cross-peaks of the peptide in the MIIbound state and thereby complicate the analysis of the NOESY spectrum, particularly when focusing on intraresidue and sequential NOE interactions. Medium and long range NOE cross-peaks are not observable prior to photo-activation at $10{ }^{\circ} \mathrm{C}$. Therefore, the background NOE data corresponding to the free peptide can be subtracted from the NOESY spectrum recorded after photo-activation. Such difference TrNOESY spectra have been used in the past for structure elucidation of antibodybound peptides, ${ }^{57}$ where spectra on separately prepared samples were subtracted from one another. Our present situation is much simpler because spectra of both the free and the transiently bound peptide can be recorded on the same sample prior to and immediately after photobleaching, respectively. NOESY spectra in the non-binding state, i.e. in the presence of darkadapted rhodopsin, and after complete decay of MII to opsin were found to be identical. For generating the NOESY difference spectrum, the dark-adapted reference spectrum was recorded immediately prior to photo-bleaching, using ten times the number of scans compared to the photo-bleached spectrum. All other parameters were identical, except for the total measuring time for the photo-bleached spectrum (38 minutes) being ten times shorter. The total time available for collection of photo-bleached TrNOE data is limited by the 54-minute decay time of the MII state.

In addition to correcting for the different number of scans, the dark-adapted spectrum was scaled by an empirical factor of 0.7 , which provided optimal suppression of the free state NOEs. This scaling factor is smaller than unity because the peptide resonances are broader and therefore weaker in the presence of the MII state. Two small regions of this TrNOE difference spectrum highlight some 

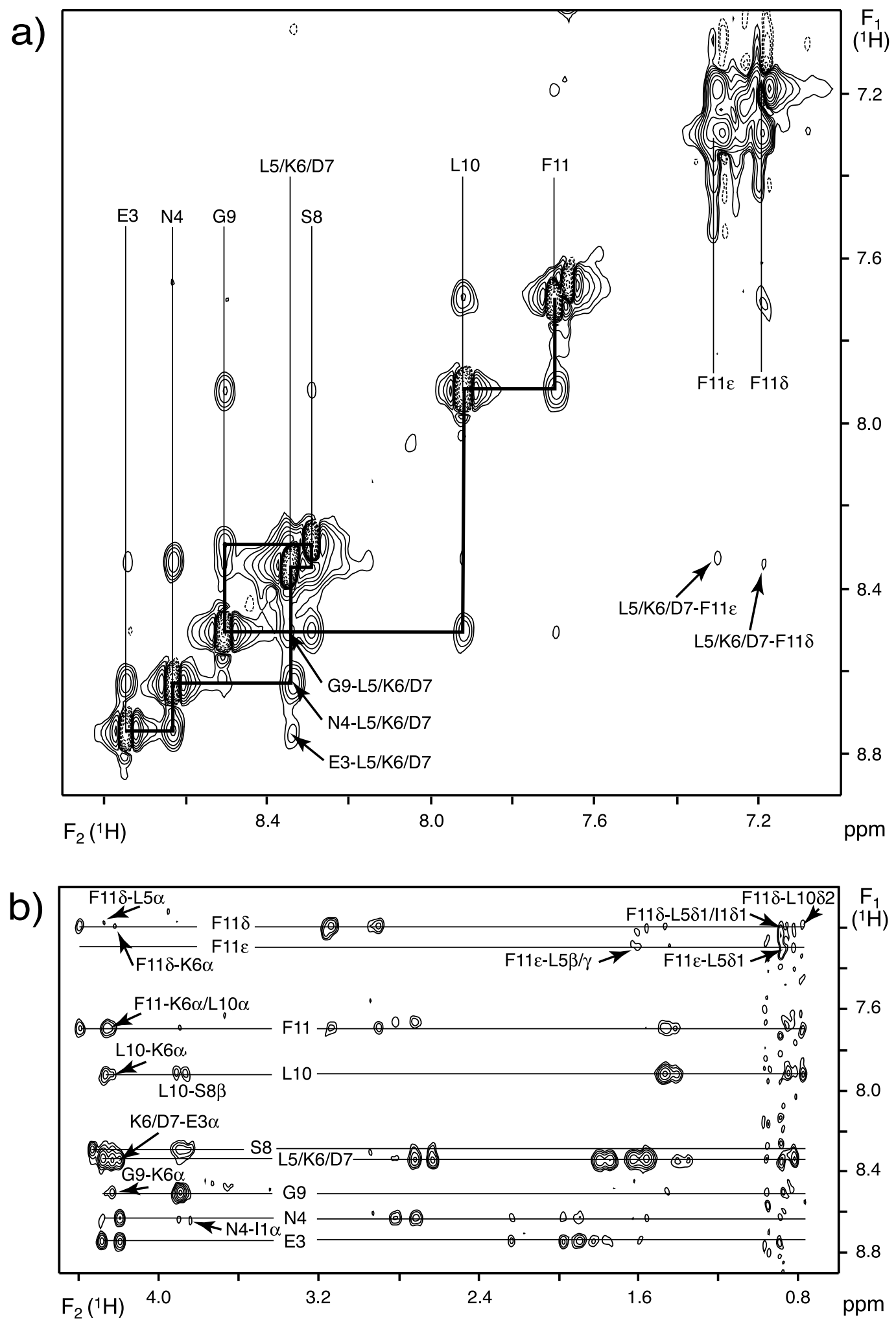

Figure 6. Small regions of the TrNOE difference spectrum of the S2 peptide. The spectrum represents the difference between spectra recorded after photo-activation of rhodopsin and a reference spectrum, recorded in the dark-adapted state. A scaling factor of 0.7 was used for the dark-adapted spectrum in order to account for broader line widths and faster decay of the diagonal resonances in the photo-activated state. (a) Amide region, with sequential connectivities marked by a continuous line. (b) Amide-to-aliphatic region. 
of the NOEs that were used to calculate the structure of the S2 peptide in the bound state (Figure 6).

The chemical shifts observed for the S2 peptide correspond to that of the unstructured peptide and considerable chemical shift degeneracy in the analysis of the NOE spectrum is therefore present, even though the spectrum was recorded at $750 \mathrm{MHz}{ }^{1} \mathrm{H}$ frequency. Resolution in the $t_{1}$ dimension of the TrNOESY spectrum is limited by the modest number of $t_{1}$ increments (200) that could be recorded prior to decay of the MII state. Moreover, with increasing duration of $t_{1}$ the decay of the MII state decreases TrNOE contributions to cross-peaks, resulting in additional cross-peak line broadening in the $F_{1}$ dimension of the 2D spectrum. Only 24 out of 121 observed peaks are uniquely assigned. Ambiguities in the other crosspeaks result either from resonance overlap or from the lack of stereospecific assignments. Even if stereospecific assignments were made for the diastereotopic methylene protons in the S2 peptide, their strong dipolar interaction causes essentially complete equilibration between their nuclear spin magnetization, preventing the possibility to separately quantify NOE interactions to the individual protons in such pairs. For the two leucine residues, stereospecific assignments of the methyl groups is made on the basis of their ${ }^{13} \mathrm{C}$ chemical shifts. Interpretation of fractional ${ }^{13} \mathrm{C}$ labeling patterns ${ }^{58}$ in denatured proteins indicates that ${ }^{13} \mathrm{C}^{\delta 1}$ resonates $1.5-2 \mathrm{ppm}$ downfield of ${ }^{13} \mathrm{C}^{\delta 2}$. ${ }^{59}$ For both leucine residues, the $\mathrm{H}^{\alpha}-\mathrm{C}^{\delta 2} \mathrm{H}_{3}$ TrNOE is considerably stronger than the $\mathrm{H}^{\alpha}-\mathrm{C}^{\delta 1} \mathrm{H}_{3}$ interaction, indicating $\chi_{2} \approx 180^{\circ}$, which automatically restricts $\chi_{1}$ to the $-60^{\circ}$ rotamer. ${ }^{60}$ Out of the total set of TrNOE restraints, seven interactions between Phe11 side-chain and Leu5 protons are the only ones classified as "longrange" $(|i-j|>5)$. In contrast to the data reported by Kisselev et al. ${ }^{20}$ no unambiguous evidence for NOE interactions between Phe11 and Ile1 was present in the TrNOESY spectrum, although the possibility of an NOE from Phe11 to Ile- $\mathrm{C}^{\delta} \mathrm{H}_{3}$ cannot be excluded a priori due to its exact overlap with Leu5- $\mathrm{C}^{\delta 1} \mathrm{H}_{3}$. Several of the backbone dihedral angles $\phi$ and $\psi$ could be restrained on the basis of the intensities of intraresidual and sequential $\mathrm{H}^{\alpha}-\mathrm{H}^{\mathrm{N}}$ NOEs. For six residues (Glu3, Asn4, Asp7, Ser8, Leu10, and Phe11) negative $\phi$ angles $\left(-90^{\circ}\left( \pm 90^{\circ}\right)\right)$ are indicated by the absence of a strong intraresidue $\mathrm{H}^{\alpha}-\mathrm{H}^{\mathrm{N}} \mathrm{NOE}$. The Ser8 $\psi$ angle is restrained to $-60^{\circ}\left( \pm 90^{\circ}\right)$ on the basis of a small sequential versus intraresidue $\mathrm{H}^{\alpha}-\mathrm{H}^{\mathrm{N}}$ NOE intensity ratio. ${ }^{61}$ Although the TrNOE data show indications of some spin diffusion, particularly for interactions to methylene proton pairs, such indirect effects are not dominating the TrNOE spectrum. For example, intraresidue $\mathrm{H}^{\epsilon}-\mathrm{H}^{\beta}$ interactions in Phe11 are more than four times weaker than $\mathrm{H}^{\delta}-\mathrm{H}^{\beta}$ cross peaks, indicating that semiquantitative analysis of the NOE cross-peaks is warranted.

\section{Structure of S2 peptide in metarhodopsin II- bound state}

No hydrogen bond restraints were used in the initial rounds of structure calculation. However, these preliminary structures were of sufficient quality that $\alpha$-helical hydrogen bonds ( $i$ to $i-4$ ) from residues Leu5-Ser8 to Ile1-Asn4 were clearly recognizable, particularly in the structures that included dipolar coupling restraints. Also, in all preliminary structures analyzed, the DSSP program $^{62}$ identifies a hydrogen bond between Gly9- $\mathrm{H}^{\mathrm{N}}$ and Leu5-CO, with Gly9 adopting a positive $\phi$ angle. This is characteristic of the $\alpha_{\mathrm{L}}$-type C-capping motif, ${ }^{63}$ identified by Kisselev et al., in the MII-bound peptide conformation. ${ }^{20}$ Signature $\mathrm{H}$-bonds of alternative helix-terminating motifs, such as the Schellman or $\mathrm{L}_{10}$ motif $^{64}\left(\mathrm{Leu} 10-\mathrm{H}^{\mathrm{N}}\right.$ to Leu5-CO and Gly9- $\mathrm{H}^{\mathrm{N}}$ to Lys6-CO), or a type II $\beta$-turn involving residues $7-10\left(\right.$ Leu10- $\mathrm{H}^{\mathrm{N}}$ to Asp7-CO) or residues 8-11 (Phe11- ${ }^{N}$ to Ser8-CO) are absent in the vast majority of analyzed structures. Introduction of such (incorrect) H-bonds as additional restraints in preliminary structure calculations led to large violations of NOEdistances and dipolar couplings.

In the final round of structure calculations, the five unambiguous hydrogen bonds from residues Leu5-Gly9 to Ile1-Leu5 were used as additional input restraints, both for the NOE-only set of calculations and for the set that includes both dipolar and NOE restraints. Analysis of all calculated structures indicates the absence of systematic violations of NOE restraints larger than $0.2 \AA$. A systematic violation of dipolar couplings by more than twice their experimental uncertainty was observed in one instance only: the ${ }^{13} \mathrm{C}^{\alpha}-{ }^{1} \mathrm{H}^{\alpha}$ coupling of Glu3, which remains violated by $1.2 \mathrm{~Hz}$. The difference in Glu3 ${ }^{13} \mathrm{C}^{\alpha}-{ }^{1} \mathrm{H}^{\alpha}$ dipolar couplings measured at $10^{\circ} \mathrm{C}(0.8 \mathrm{~Hz})$ and $20^{\circ} \mathrm{C}$ $(3.1 \mathrm{~Hz})$ is larger than for any other coupling, and a larger-than-average measurement error likely contributes to its poorer fit to the final structure. The magnitude of the dipolar coupling violations is inversely related to the size of the force constant used in the structure calculations. In order to account for potential differences in local dynamics, this force constant was intentionally kept weak, enforcing the dipolar coupling restraints to a lesser degree than warranted by the experimental uncertainties.

In order to evaluate the effect of the dipolar couplings on the final structures, a control set was calculated without dipolar coupling restraints. The 20 models with lowest energy from each set show a high degree of similarity (Figure 7; Table 1). This high similarity is slightly deceptive for two reasons. First, hydrogen bonds inferred from preliminary structure calculations without $\mathrm{H}$-bond restraints were much clearer in the structures that included dipolar restraints (see above). However, identical sets of $\mathrm{H}$-bond restraints were used in the calculations of the final structures with and 

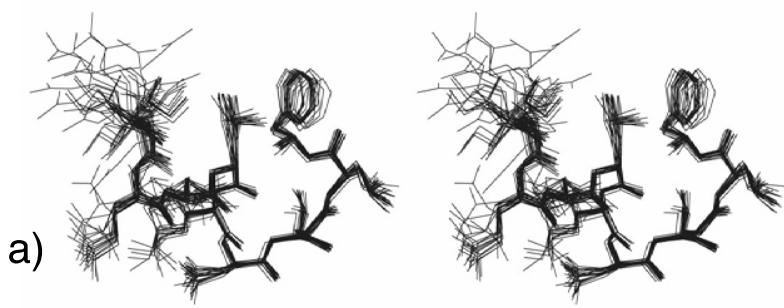

b)
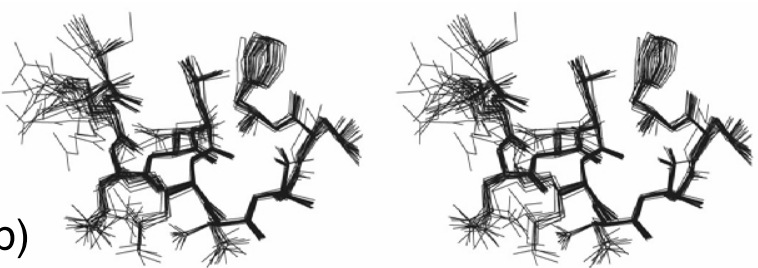

c)
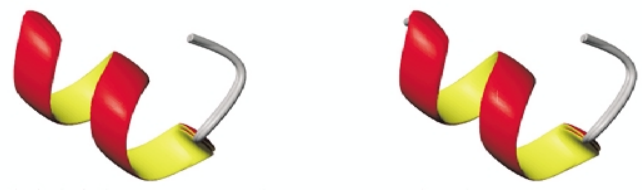

Figure 7. Stereodiagrams of the set of 20 lowest energy structures of the S2 peptide in the MII bound state, calculated (a) with and (b) without dipolar couplings. (c) Superposition of the two average structures from (a) and (b), shown as backbone ribbon diagrams, showing that the backbones of the two families of structures are virtually indistinguishable. For orientation of peptide relative to membrane normal, see Figure 11.

without dipolar couplings. Second, a number of NOE restraints were re-evaluated only after they were found to be incompatible with dipolar restraints. For example, in the initial rounds of structure calculations, error margins of the NOEderived distance restraints were used that turned out to be too tight. With such tight restraints, NOE and dipolar coupling restraints could not be satisfied simultaneously. Enforcing better agreement by using higher force constants resulted in unduly restrained conformations, as indicated by a low backbone rmsd of the set. Moreover, several residues, most notably Arg2, clustered in disallowed regions of the Ramachandran plot. Only after the NOE distance bounds were relaxed according to the empirical rules given in Materials and Methods, could both NOE and residual dipolar coupling restraints be satisfied simultaneously, and the Ramachandran score improved significantly. Naturally, the new structures were less tightly defined than the initial set, reflected by a higher backbone rmsd. Two clusters of structures were observed in the new set, differing in the conformation of the C-terminal Phe11 side-chain. However, a unique conformation was obtained
Table 1. Summary of structural statistics

\begin{tabular}{|c|c|c|}
\hline & $\begin{array}{l}\text { Without } \\
\text { dipolar } \\
\text { couplings }\langle\mathrm{SA}\rangle^{\mathbf{a}}\end{array}$ & $\begin{array}{l}\text { With dipolar } \\
\text { couplings } \\
\left\langle\mathrm{SA}_{\text {dipolar }}\right\rangle^{\mathrm{a}}\end{array}$ \\
\hline \multicolumn{3}{|c|}{ RMSDs from experimental distance restraints $(\AA)$} \\
\hline All $(121)^{\mathbf{b}}$ & 0.024 & 0.042 \\
\hline Intraresidue (44) & 0.028 & 0.039 \\
\hline $\begin{array}{l}\text { Interresidue sequential } \\
(|i-j|=1)(33)\end{array}$ & 0.030 & 0.045 \\
\hline $\begin{array}{l}\text { Interresidue medium range } \\
(1<|i-j| \leq 5)(37)\end{array}$ & 0.011 & 0.042 \\
\hline $\begin{array}{l}\text { Interresidue long range } \\
(|i-j|>5)(7)\end{array}$ & 0.005 & 0.009 \\
\hline \multicolumn{3}{|c|}{ RMSDs from residual dipolar couplings $(\mathrm{Hz})$} \\
\hline${ }^{1} D_{\mathrm{NH}}(9)$ & 0.48 & 0.15 \\
\hline${ }^{1} D_{\mathrm{C} \alpha \mathrm{H} \alpha}(9)$ & 0.65 & 0.49 \\
\hline${ }^{1} D_{\text {sidechain }{ }_{\mathrm{CH}}(20)}$ & 1.58 & 0.07 \\
\hline \multicolumn{3}{|c|}{ RMSDs from experimental dihedral restraints $\left({ }^{\circ}\right)$} \\
\hline NOE based dihedrals (12) & 0 & 0 \\
\hline \multicolumn{3}{|c|}{ Deviations from idealized covalent geometry } \\
\hline Bonds (@) (185) & 0.0012 & 0.0024 \\
\hline Angles $\left({ }^{\circ}\right)(334)$ & 0.15 & 0.39 \\
\hline Impropers $\left({ }^{\circ}\right)(93)$ & 0.18 & 0.37 \\
\hline \multicolumn{3}{|c|}{ Structure quality (Ramachandran plot) } \\
\hline $\begin{array}{l}\text { Residues in most favored } \\
\text { region }(\%)\end{array}$ & 88 & 100 \\
\hline $\begin{array}{l}\text { Residues in additionally } \\
\text { allowed regions }(\%) \\
\text { Coordinate precision }(\AA)^{\mathrm{d}}\end{array}$ & 12 & 0 \\
\hline Backbone atoms $\left(\mathrm{N}, \mathrm{C}_{\alpha}, \mathrm{C}^{\prime}, \mathrm{O}\right)$ & 0.15 & 0.17 \\
\hline All non-hydrogen atoms & 0.86 & 0.98 \\
\hline
\end{tabular}

a $\langle\mathrm{SA}\rangle$ and $\left\langle\mathrm{SA}_{\text {dipolar }}\right\rangle$ are averages of 20 simulated annealing structures with lowest total energy out of 100 calculated without and with dipolar coupling restraints, respectively.

b Numbers between brackets are the number of restraints.

c Non-glycine residues; excluding end-residues.

d Defined as the average rms difference (residues 2-11) between the final 20 simulated annealing structures and the mean coordinates.

after adding lower limits to the NOE table for decisive proton-proton distances for which the absence of an NOE could be clearly established.

A stereo-diagram of the energy-minimized average structure of the S2 peptide (Figure 8)
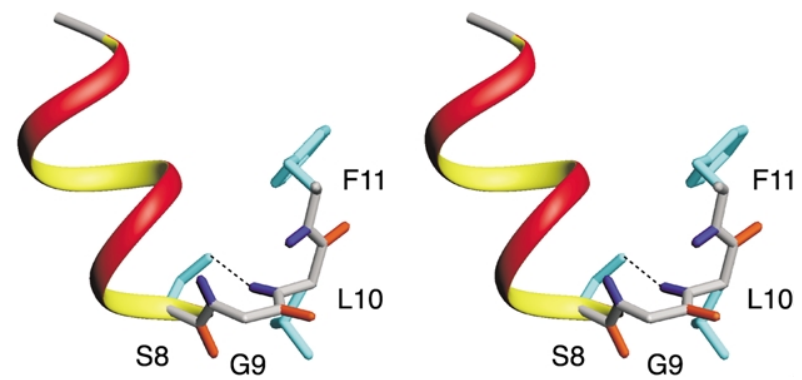

Figure 8. Stereodiagram of the energy-minimized average structure of the S2 peptide in the MII-bound state. The $\mathrm{N}$-terminal eight residues are represented by an $\alpha$-helical ribbon. The C-terminal reverse open turn residues are shown as stick representation, with backbone $\mathrm{NH}$ and $\mathrm{CO}$ bonds in blue and red, respectively, and side-chains in cyan. Close proximity $(2.1 \AA)$ of the Ser8- $\mathrm{O}^{\gamma}$ and Leu10- $\mathrm{H}^{\mathrm{N}}$ indicate a stabilizing hydrogen bond (broken line). The program MOLMOL ${ }^{95}$ was used for making this Figure. 
shows that the hydrophobic Leu10 side-chain points away from the remainder of the peptide, which would result in very unfavorable exposure to solvent if this structure were present in a free state in solution. The structure also shows the presence of a short $(2.1 \AA)$ distance between Leu10- $\mathrm{H}^{\mathrm{N}}$ and Ser8-O ${ }^{\gamma}$, indicative of a second $\mathrm{H}$-bond (in addition to the Gly9- $\mathrm{H}^{\mathrm{N}}$ to Leu5-CO $\mathrm{H}$-bond) stabilizing the $\alpha_{\mathrm{L}} \mathrm{C}$-cap (Figure 8). It is also interesting to note that the backbone near the C terminus forms a rather "open" structure, in which both the amide proton of Phe11 and the carbonyl oxygen atoms of Lys6 to Phe11 are able to donate and accept hydrogen bonds. From an energetic perspective, such hydrogen bonding is essential upon complex formation when shielded from the aqueous solvent.

At the relatively weak dipolar force constants used, the precision of the structure with dipolar couplings included (backbone rmsd $=0.17 \AA$ ) is virtually the same as for the NOE-only structure (backbone rmsd $=0.15 \AA$ ) (Table 1 ). However, the quality of the backbone structures as evaluated by the program PROCHECK ${ }^{65}$ is better for the structure that includes the dipolar couplings $(100 \%$ of the residues in the most-favored region of the Ramachandran map) than for the NOE-only structure $(88 \%)$.

\section{Peptide orientation and rhodopsin contacts}

Refinement against residual dipolar couplings provides the mean angle, $\beta=40^{\circ}( \pm 4)^{\circ}$, between the helix axis of the MII-bound S2 peptide and the membrane normal. On the $\alpha$-helical cylinder, the face marked by Leu5 and Ser8 $\mathrm{C}^{\alpha}$ atoms is closest to the membrane. However, as discussed later, this orientation does not imply that this is the surface making most contacts with MII.

The twofold degeneracy of dipolar couplings with respect to the angle $\theta$ (equations (3)) results in an ambiguity for the vectorial orientation of the peptide, i.e. which end of the peptide points towards rhodopsin. However, this ambiguity is resolved by considering that the first eight residues of the S2 peptide form an extension of the crystallographically identified C-terminal helix of $\mathrm{G}_{\mathrm{t}} \alpha$, which means that for steric reasons it is the C-terminal end of the peptide which must be pointing towards rhodopsin. As discussed below, this is confirmed by the TrNOESY cross-relaxation data which contain information on magnetization transfer between rhodopsin and the S2 peptide.

The NMR spectrum of rhodopsin, anchored in the lipid bilayer, is extremely broad and is not effectively excited by the radiofrequency pulses used in the TrNOE experiment. As a result, magnetization can flow from the peptide to rhodopsin during the NOE mixing time of the experiment, but the inverse process does not take place. Magnetization flow from a peptide proton, X, to rhodopsin will decrease the intensity of magnetization observed during the detection time that is

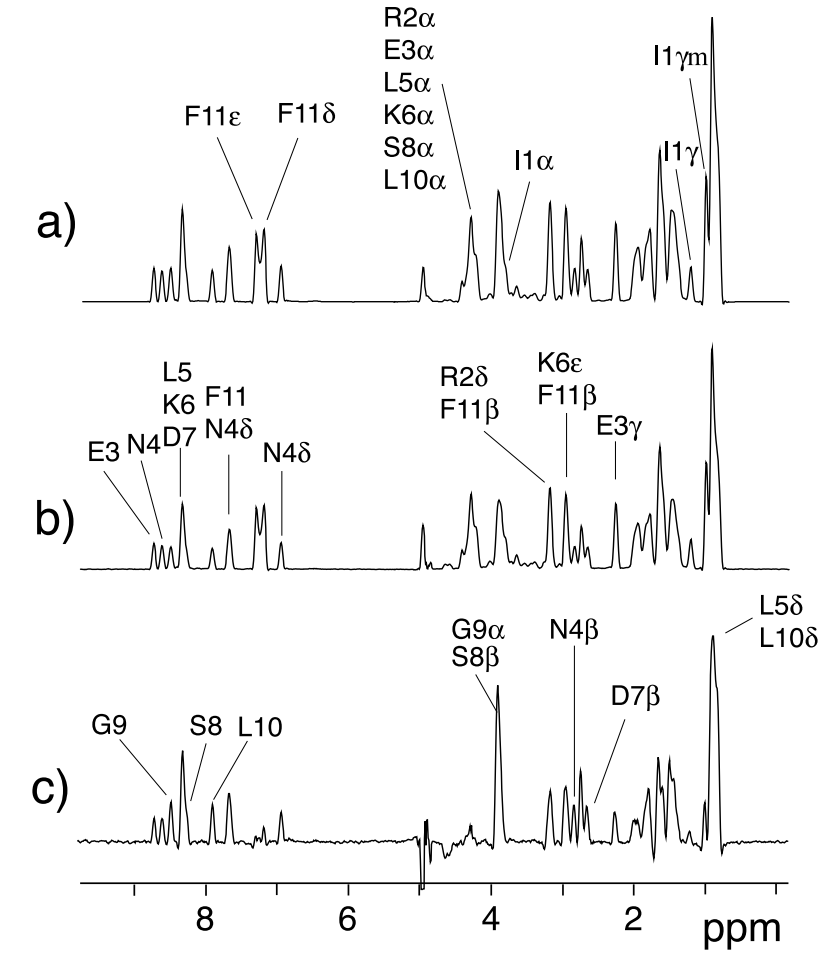

Figure 9. Projections onto the $F_{1}$ axis of the NOESY spectra of S2 peptide recorded (a) prior to and (b) after photo-generation of the peptide-binding MII state of rhodopsin. (c) Difference spectrum, scaled up by a factor 2.5. Faster transverse relaxation after photo-bleaching during a $4 \mathrm{~ms}$ WATERGATE delay, needed for water suppression, was empirically accounted for by a 1.1 scaling factor of the bleached spectrum (b), prior to calculating the difference spectrum (c). The $6-4.45 \mathrm{ppm}$ band, which contains $t_{1}$ noise from the residual $\mathrm{H}_{2} \mathrm{O}$ signal, is not included in the integral-projection. Assignments of the least attenuated, medium attenuated and most attenuated resonances are marked in (a), (b), and (c), respectively.

modulated by $\omega_{x}$, the frequency of proton $X$. Comparison of the integrated signal intensity, projected onto the $F_{1}$ axis of the 2D NOESY spectrum, in the dark and photo-activated states therefore identifies protons which have magnetization "leaking" to rhodopsin (Figure 9). Although resonance overlap in the one-dimensional projected spectrum prohibits measurement of the attenuation ratio for many individual resonances, a number of hydrogen atoms can be identified that clearly are strongly attenuated. Most attenuated are the amide protons of Gly9 and Leu10, which are on the outside of the $\alpha_{\mathrm{L}}$ turn and are inserted deepest into rhodopsin. Considerable attenuation is also seen for the side-chain protons of Asn4 and Asp7 and for the overlapping Ser8- $\mathrm{H}^{\beta}$ and Gly $9-\mathrm{H}^{\alpha}$ resonances, indicating their proximity to hydrogen atoms on the rhodopsin surface. Relatively little (11\%) attenuation is observed for the aromatic protons on the C-terminal residue, indicating the absence of tight hydrophobic packing of the aromatic ring against rhodopsin 
residues. The Ile1- $\mathrm{H}^{\alpha}$ resonance is visible as the upfield shoulder of the overlapping Ser8- $\mathrm{H}^{\beta}$ and Gly $9-\mathrm{H}^{\alpha}$ resonances, and shows little attenuation. As expected, the $\mathrm{N}$-terminal Ile1- $\mathrm{C}^{\gamma} \mathrm{H}_{3}$ resonance shows the weakest attenuation (4\%) of all, indicating that the N-terminal end of the peptide does not make any direct contacts with rhodopsin.

\section{Discussion}

Transferred dipolar couplings provide very useful restraints for studying the conformation of a ligand when bound to its target, which has been aligned with respect to the magnetic field. These dipolar couplings complement TrNOE data by providing information on both the relative orientation of internuclear vectors within the ligand, and on the orientation of the ligand relative to its aligned target. Similar to the TrNOE experiment, measurements are easiest when the ligand is in fast exchange between the free and the bound state, i.e. only a single set of resonances is observable.

The peptide sequence used here, IRENLKDSGLF, differs from $\mathrm{G}_{t} \alpha(340-350)$ by two substitutions. Replacement K341R was applied for the sake of compatibility with a previous TrNOE study of the $G_{t} \alpha(340-350)-K 341 R$ peptide. ${ }^{19}$ As judged by the about sevenfold decrease of $\mathrm{EC}_{50}$ in dose-response curves, the K341R substitution increases the potency of the peptide to interact with and stabilize MII. ${ }^{18}$ The second substitution (C347S) reduces the affinity of the peptide to MII, and avoids the need for adding reducing agents to the sample. Screening of a combinatorial library of $\mathrm{G}_{t} \alpha(340-350)$ peptide analogs for high affinity to MII yielded exclusively 11-mer peptides with Cys in position 8 during the final rounds of panning. ${ }^{66}$ However, acetylated $G_{t} \alpha(340-350)-K 341 R-C 347 S$ is able to bind and stabilize MII, albeit with lower affinity and potency. ${ }^{18}$

\section{Magnetic alignment of disk membranes}

Natural, intact retinal rods are known to align strongly with the magnetic field. ${ }^{41}$ These rods consist of highly ordered axial stacks of disk membranes, which are essentially extremely flattened vesicles. Rhodopsin with its seven transmembrane helices accounts for about $50 \mathrm{wt} \%$ of the disk membrane and is by far its major protein component. A small fraction of rim protein is preferentially located along the edge region. ${ }^{67}$ Lipids account for most of the remaining $\sim 50 \mathrm{wt} \%$ of the disks. The positive magnetic susceptibility anisotropy of rhodopsin's transmembrane helices ${ }^{68}$ and their near-parallel alignment throughout the entire retinal rod form a strong driving force for alignment of the rods when placed in a magnetic field. ${ }^{41}$ Individual rod outer segment disks lack the cooperativity in alignment that exists for intact rods, but have a sufficiently large total magnetic susceptibility anisotropy to align relative to the magnetic field. ${ }^{43}$ The degree of alignment is essentially the same at NMR field strengths of $14.2 \mathrm{~T}$ and $18.8 \mathrm{~T}$, as monitored by a field-independent ${ }^{2} \mathrm{H}$ quadrupole splitting of ca $2 \mathrm{~Hz}$ for the lock solvent signal $\left(20 \mathrm{mg}\right.$ disks $/ \mathrm{ml}$ at $\left.20^{\circ} \mathrm{C}\right)$. Also, as shown previously, ${ }^{43}$ the peptide dipolar couplings at the two field strengths are of very similar magnitude. This indicates that the magnetic alignment of the disks is essentially saturated.

\section{Metarhodopsin II induces conformation of $G_{t} \alpha$ C terminus}

In the resting state, the $\alpha$-subunit, $G_{t} \alpha$, of the heterotrimeric $G$ protein transducin, $G_{t} \alpha \beta \gamma$, binds GDP. Light activation of rhodopsin causes a conformational change of the $G$ protein receptor that triggers binding of $G_{t} \alpha \beta \gamma \cdot G D P$. The activated receptor catalyses a GDP for GTP exchange on $G_{t} \alpha \beta \gamma$, followed by separation of $G_{t} \alpha \cdot G T P$ from both the receptor and the $G_{t} \beta \gamma$ heterodimer. X-ray structures of $\mathrm{G}_{\mathrm{t}} \alpha \cdot \mathrm{GTP}, \mathrm{G}^{69} \mathrm{G}_{\mathrm{t}} \alpha \cdot \mathrm{GDP},{ }^{70}$ and $\mathrm{G}_{\mathrm{t}} \alpha \beta \gamma \cdot \mathrm{GDP}, 7$ all crystallized in the absence of rhodopsin, indicate a disordered $C$ terminus of $G_{t} \alpha$, independent of the type of nucleotide bound. Of the 11 C-terminal residues of $G_{t} \alpha$, residues 340-342 are part of $\alpha$-helix 5, but no electron density is observed for residues 343-350. In contrast, two NMR studies on the C-terminal undecapeptide of $G_{t} \alpha$ indicate a distinct conformation for the peptide in the receptor-bound state. ${ }^{19,20}$ In the pioneering work by Dratz et al., the transferred NOE technique was used to study the bound peptide prior to and after photo-activation of the receptor. Reported structural features of the MIIbound peptide include a relaxed helical turn at the $\mathrm{N}$ terminus, and a hydrophobic cluster close to the $\mathrm{C}$ terminus, that is thought to be critical for binding. The low signal-to-noise ratio of the NOESY spectrum recorded after light activation and the limited number of NOE distance restraints obtainable at the lower magnetic field strength, as well as indirect magnetization transfer effects via receptor protons, ${ }^{71,72}$ prevented the authors from deriving a detailed molecular model for the bound peptide.

A subsequent TrNOE study by Kisselev et al. detailed the NMR structure of the MII-bound $G_{t} \alpha(340-350)$ peptide, and showed that $G_{t} \alpha(340-$ 350) is largely unstructured in the presence of dark-adapted rhodopsin. ${ }^{20}$ Kisselev et al. observed an $\alpha$-helical geometry for residues $342-346$ in the MII-bound peptide, followed by an open reverse turn centered at Gly348, that was classified as an $\alpha_{\mathrm{L}}$-type helix capping motif on the basis of an H-bond from Gly348-H ${ }^{N}$ to Leu344-CO and a left-hand conformation of Gly348. ${ }^{20}$ The general features of our MII-bound S2 peptide presented here agree well with this $G_{t} \alpha(340-350)$ structure (Figure 10), despite the two K2R and C8S substitutions used in our work. However, the N-terminal helix is longer and more clearly defined in S2, with $\alpha$-helical hydrogen bonds from 

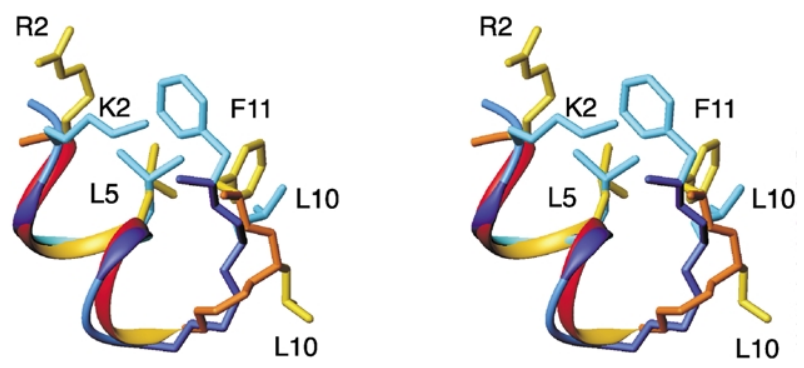

Figure 10. Superimposed MII-bound structures of the S2 (red/gold) and $\mathrm{G}_{\mathrm{t}} \alpha(340-350)$ (blue/cyan) ${ }^{20}$ peptides. The N-terminal eight residues are shown as ribbon representation, the backbone-bonds of the remaining three residues and bonds connecting non-hydrogen atoms in selected side-chains are shown as sticks. The helix extends from residue 2 to 8 in S2, and from 3 to 6 in $\mathrm{G}_{\mathrm{t}} \alpha(340-350)$. The fit of the two structures is on the basis of minimizing the rms difference of the backbone atom positions of residues 3-6 only.

Leu5-Ser8 to Ile1-Asn4. A number of signature $\alpha$-helical distance constraints extracted from the S2 peptide TrNOE data are violated in the published $\mathrm{G}_{t} \alpha(340-350)$ structures. This results in a rather large rms difference of $0.7 \AA$ between the backbone atom coordinates of residues $1-5$ of the energy minimized mean structures of the S2 and $\mathrm{G}_{\mathrm{t}} \alpha(340-350)$ peptides.

The glycine-based $\alpha_{\mathrm{L}}$-type helix capping motif, first identified by Kisselev et al., in the $\mathrm{G}_{\mathrm{t}} \alpha(340-350)$ peptide, is also clearly visible in the MII-bound S2 peptide. An additional $\mathrm{H}$-bond between Leu10- $\mathrm{H}^{\mathrm{N}}$ and Ser8-O $\mathrm{O}^{\gamma}$ is observed in the S2 peptide, which presumably serves to stabilize this turn. Screening of a combinatorial library of peptide analogs to $G_{t} \alpha(340-350)$ indicated that Gly348 is essential for binding to MII. ${ }^{66}$ Statistical analysis shows that glycine-based motifs are prevalent at the $\mathrm{COOH}$ termini of $\alpha$-helices ${ }^{73}$ where Gly most frequently occurs in the $C^{\prime}$ position, i.e. adopting a left-hand conformation and forming an $\mathrm{H}$-bond between the amide proton of Gly and the backbone $\mathrm{CO}$ of residue $i-4$ ( $\alpha_{\mathrm{L}}$-motif) or $i-3$ (L3 10 -motif). ${ }^{63}$

Small differences between the bound $\mathrm{G}_{\mathrm{t}} \alpha(340-350)$ and $\mathrm{S} 2$ peptides are seen for many of the side-chains. TrNOE data for the S2 peptide lack evidence for close proximity of the Phe11 aromatic ring and the side-chains of residues 1 and 2 , seen in the TrNOESY of $G_{t} \alpha(340-350){ }^{20}$ As a consequence, the Lys341 side-chain in $\mathrm{G}_{\mathrm{t}} \alpha(340-350)$ is much closer to the C-terminal Phe aromatic group than the Arg2 side-chain in the S2 structure (Figure 10). In the S2 peptide structure, the Phe11- $\mathrm{C}^{\beta} \mathrm{H}_{2}$ protons are closer to Lys6- $\mathrm{H}^{\alpha}$ and Leu5- $\mathrm{H}^{\beta} / \mathrm{H}^{\gamma}$, as indicated by NOE distances of $3.0( \pm 0.8) \AA$ and $3.3( \pm 1) \AA$, respectively, which are systematically shorter than the corresponding distances in the $G_{t} \alpha(340-350)$ structure. The sidechains of residues Leu5, Leu10, and Phe11, which are highly conserved among G-proteins, form a hydrophobic cluster in $G_{t} \alpha(340-350)$, whereas in the S2 structure, Leu10 makes no direct contacts with either of the other two side-chains.

The observed differences between the $G_{t} \alpha(340-$ 350 ) and S2 peptide conformations may result in part from the two conservative substitutions in S2. However, experimental factors may also play a role. The weaker binding affinity of S2 reduces the

Table 2. Simulated annealing protocol

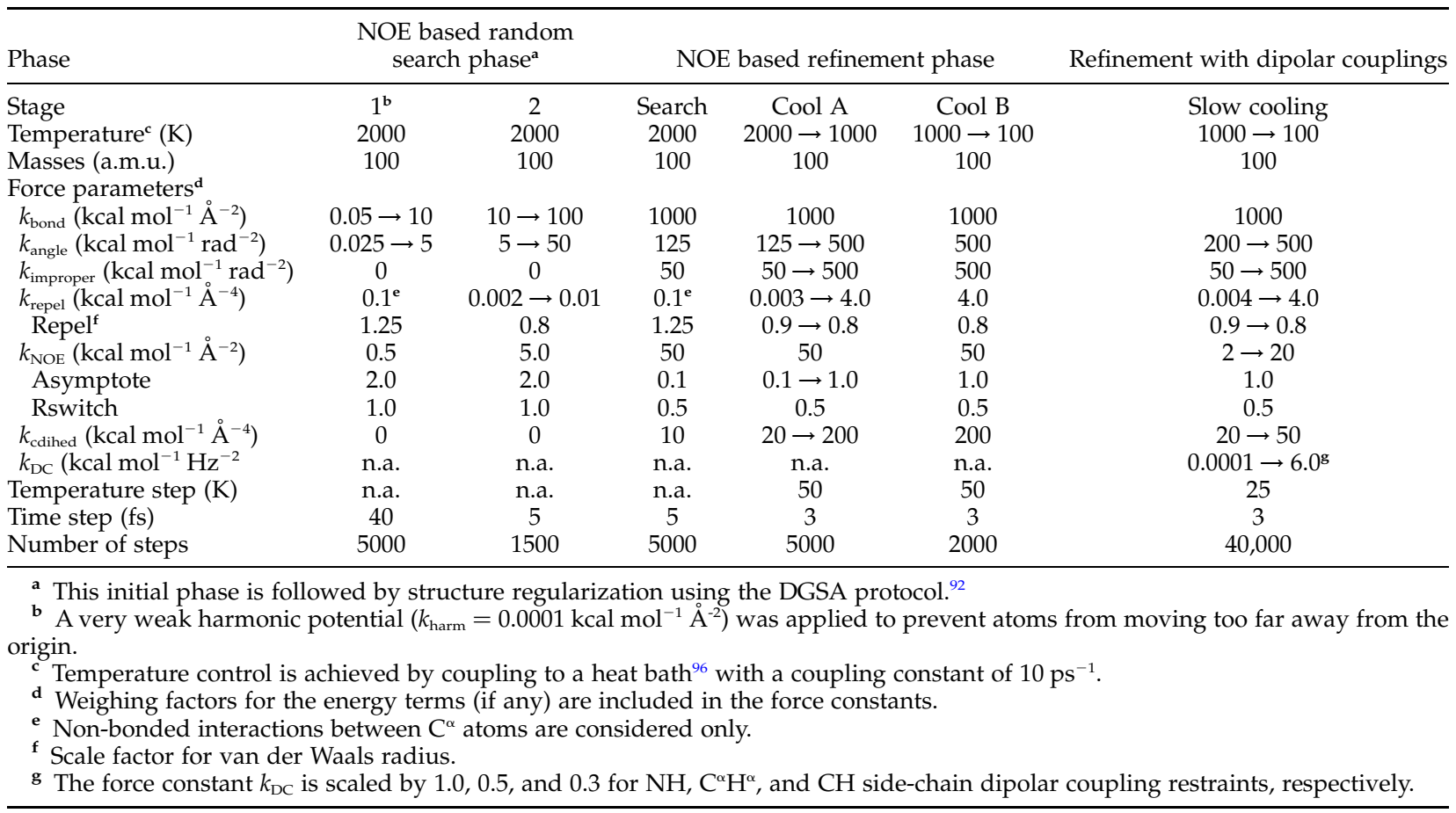


risk of indirect magnetization transfer during a single bound cycle, and the higher magnetic field strength available in our study facilitated the recording of well resolved NOESY spectra at relatively short mixing times. Most importantly, however, the use of residual dipolar couplings in the S2 structure calculation further diminishes the influence of potentially biased distance restraints. The S2 structure shows all pairs of backbone $\phi / \psi$ torsion angles in the most-favored region of the Ramachandran map (Table 1), confirming that the structure is physically reasonable. ${ }^{74}$

The absence of electron density for the $G_{t} \alpha$ C-terminal residues in the crystal structure contrasts with the well defined NMR structure of the MII-bound $G_{t} \alpha(340-350)$. This result indicates that extension of helix 5 and its capping with the $\alpha_{\mathrm{L}}$ helix-terminating motif are stabilized by specific interactions between peptide and photo-activated rhodopsin.

Statistical analysis of C-terminal helix capping motifs with Gly in the $C^{\prime}$ position indicates that the polarity of the residue in the $C^{\prime \prime}$ position of the capping motif usually determines whether a $\mathrm{L}_{10}$ or a $\alpha_{\mathrm{L}}$-motif is adopted, with an apolar residue in $C^{\prime \prime}$ (Leu10 in S2 peptide) predictive for a L3 $_{10}$-motif. ${ }^{63}$ The observation of an $\alpha_{\mathrm{L}}$-motif in rhodopsin-bound $\mathrm{S} 2$ is counter to this rule. In the absence of rhodopsin, exposure to solvent of the Leu10 side-chain in the $\alpha_{\mathrm{L}}$ type C-cap is energetically unfavorable and explains the unstructured nature of the $C$ terminus in the X-ray structure. Kisselev et al. proposed that the sidechain of the leucine in the $C^{\prime \prime}$ position is part of a very hydrophobic patch on the peptide surface, which they suggest to constitute a significant portion of the rhodopsin-binding surface. ${ }^{20}$ Interestingly, the structure of the last three residues is relatively "open" (Figure 8) with all backbone carbonyl groups of Lys6-Phe11 and the backbone amide of Phe11 accessible to form hydrogen bonds with rhodopsin. Analysis of the intermolecular magnetization transfer (Figure 9) indicates that, in addition to Gly9 and Leu10, the side-chains of Asn4 and Asp7 are also in close proximity to rhodopsin protons.

A recent cross-linking study indicates spatial proximity between Ser240 in the third cytoplasmic loop of light-activated rhodopsin and the amino acid sequence $342-345$ of $G_{t} \alpha{ }^{75}$ which corresponds to Glu3-Lys6 in the S2 peptide, thus confirming the close contact between the helical region of the S2 peptide and MII. In earlier studies, helix 8 of rhodopsin (previously referred to as the fourth cytoplasmic loop) has also been implicated in binding to $\mathrm{G}_{\mathrm{t}} \alpha(340-350){ }^{76}$

\section{Towards a model for the complex}

The bound S2 peptide conformation was docked to the $2.0 \AA$ crystal structure of $G_{t} \alpha \beta \gamma$.GDP (PDB accession code 1GOT) by best fitting the coordinates of the backbone atoms $G_{t} \alpha(341-342)$ and S2 residues Arg2 and Glu3 in the overlapping helical stretch. The dipolar coupling data indicate that the extended helix 5 of $\mathrm{G}_{\mathrm{t}} \alpha$ makes an angle of $40^{\circ}( \pm 4)^{\circ}$ with the bilayer normal. The accuracy of the S2 peptide orientation is quite high, and the rotation of the peptide relative to the helical axis is also narrowly defined. However, docking to the 1GOT structure presumes that no sharp kink occurs in the section of helix 5 that precedes Glu342. Moreover, it is conceivable that a considerable rearrangement could occur within $\mathrm{G}_{\mathrm{t}} \alpha \beta \gamma \cdot \mathrm{GDP}$ upon binding rhodopsin. Assuming that neither of these two possibilities apply, a model for the transducin-rhodopsin complex can be generated in which there remains only one rotational degree of freedom for $G_{t} \alpha \beta \gamma \cdot G D P$, around the membrane normal (Figure 11). The orientation of $G_{t} \alpha \beta \gamma \cdot G D P$ relative to the membrane normal is rather surprising as it indicates that many of the previously identified contact sites on $\mathrm{G}_{\mathrm{t}} \alpha \beta \gamma$.GDP are not located at the bottom portion of the protein. Instead, in the oriented $G_{t} \alpha \beta \gamma$.GDP these contact sites are found on a face which spans from $G_{t} \gamma(60-71)$ at the bottom tip to the $\mathrm{G}_{\mathrm{t}} \alpha(311-328)$ strand/loop which is located substantially higher, and with the $G_{t} \alpha C$ terminus located roughly halfway in between (Figure 11). In order to accommodate all these interactions simultaneously, the $G_{t} \gamma(60-71)$ must insert itself into rhodopsin, and one or more of the previously identified rhodopsin surface loops must move away from the membrane bilayer such that contact to the $G_{t} \alpha(311-328)$ region can be made.

To date, only the structure of dark-adapted rhodopsin is known. Clearly, considerable rearrangement of the rhodopsin surface will be necessary to make the previously identified contacts when $\mathrm{G}_{t} \alpha \beta \gamma \cdot G D P$ must be kept at the orientation shown in Figure 11. The nature of this light-induced conformational change is not yet known. However, rigid body movement of transmembrane helices of rhodopsin was found to be critical for light activation by EPR spectroscopy. ${ }^{8}$ A preliminary outline for the surface of photo-activated rhodopsin recently has been generated on the basis of solution NMR, ${ }^{77}$ and current work is aimed at finding a selfconsistent model for the complex. Alternatively, a two-site sequential fit model was proposed for the signal transfer from MII to transducin, i.e. the various interaction sites do not necessarily need to come in contact simultaneously. ${ }^{4}$

As demonstrated in our study, transferred residual dipolar couplings from weakly binding ligands of membrane proteins do not only provide useful restraints for structure refinement of the bound ligand but also define the orientation of the ligand relative to the bilayer normal. The combination of dipolar couplings with distance restraints from transferred NOEs and qualitative analysis of cross-relaxation behavior defines the structure of the bound ligand and points to the contact sites on the surface of the ligand. The aim of the current study was to demonstrate the feasibility 

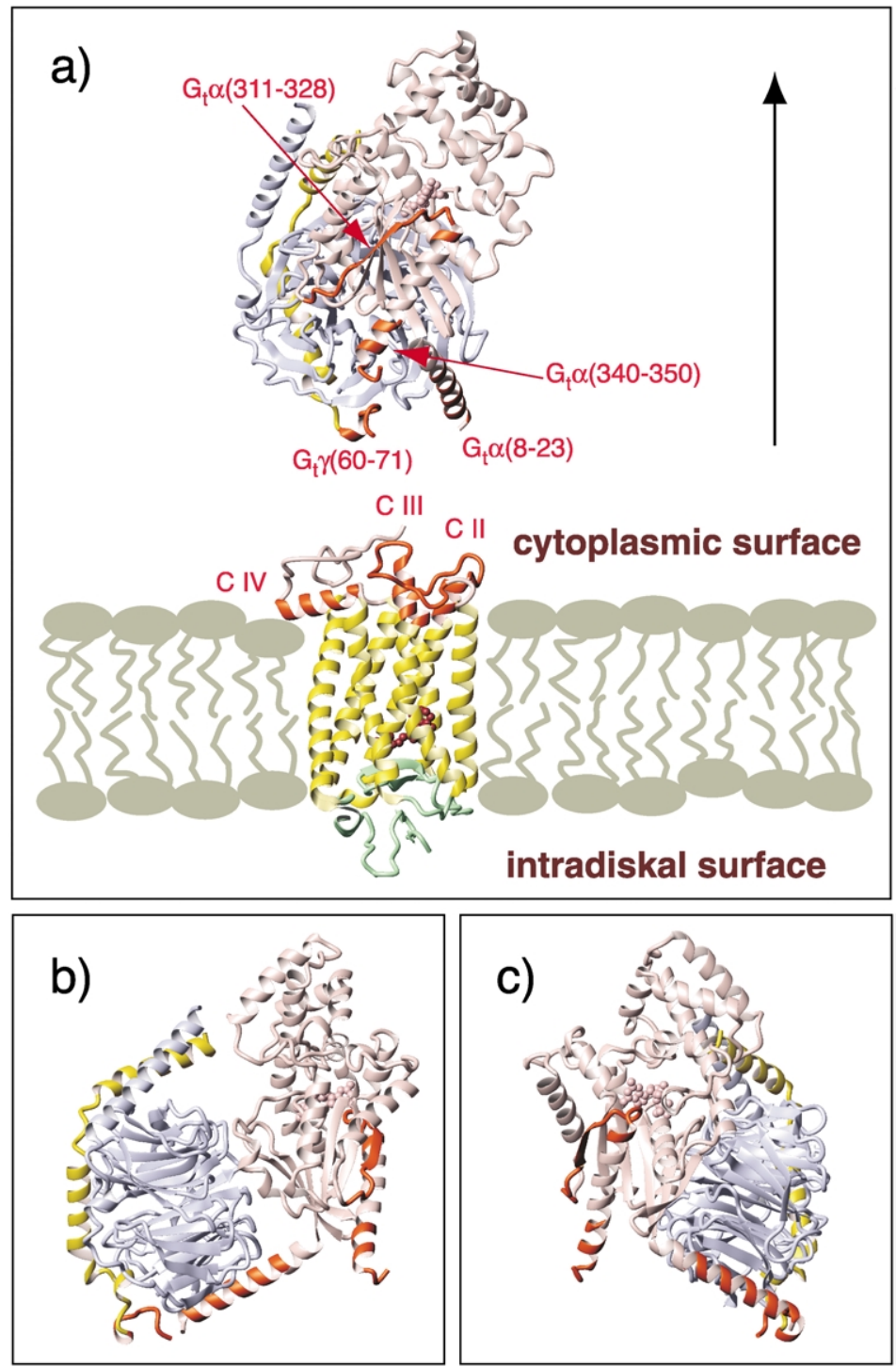

Figure 11. Schematic representation of metarhodopsin II transducin interaction (a). Ribbon diagrams are on the basis of the X-ray structures of ground-state rhodopsin ${ }^{6}$ and GDP bound transducin. ${ }^{7}$ The $C$ terminus of $G_{t} \alpha$ is not ordered in the crystal structure but $G_{t} \alpha(340-350)$ is represented by the NMR structure of the MII-bound S2 peptide that has been docked to the crystal structure. The orientation of the bound peptide relative to the membrane normal (arrow) is determined by the measured dipolar couplings, leaving just one rotational degree of freedom (rotation around the membrane normal) for the relative orientation of MII and $\mathrm{G}_{\mathrm{t}} \alpha \beta \gamma \cdot \mathrm{GDP}$. Color coded in red are potential binding regions on both proteins. Three cytoplasmic loops of rho$\operatorname{dopsin}^{76,78,79}$ and four distinct regions of transducin ${ }^{16,17}$ are likely binding sites according to mutational assays and peptide studies. (b) and (c) S2/G $\alpha \beta \gamma \cdot G D P$ models rotated by (b) $-90^{\circ}$ and (c) $60^{\circ}$ around the membrane normal relative to the structure shown in (a).

of this approach by analyzing the MII-bound conformation of the S2 peptide. Investigation of additional binding sites is likely to result in a more detailed picture of the large $\mathrm{MII} /$ transducin complex. There are several peptide candidates for such studies: a second peptide analog from the C-terminal region of the $\alpha$-subunit of transducin, $\mathrm{G}_{\mathrm{t}} \alpha(311-$ $328),{ }^{16}$ as well as a farnesylated peptide analog from the $\gamma$-subunit, $G_{t} \gamma(50-71 \text { farnesyl })^{4}$ have been shown to compete with transducin for binding to rhodopsin and stabilize MII. The $\mathrm{G}_{\mathrm{t}} \alpha(8-23)$ peptide analog also competes with transducin for binding, but without stabilizing MII. ${ }^{16}$ Potential transducin binding sites on MII include cytoplasmic loops II and III and helix 8 (Figure 11). ${ }^{76,78,79}$

\section{Materials and Methods}

\section{Peptide expression and purification}

The S2 peptide, IRENLKDSGLF, was expressed in Escherichia coli as a C-terminal extension of the immuno- globulin-binding domain of streptococcal protein $G$ (GB1 domain). DNA encoding the peptide including a flanking N-terminal linker region for FXa specificity was cloned into the GEV2 vector ${ }^{80}$ using $5^{\prime}$ Bam HI and $3^{\prime}$ Xho I restriction sites. BL21 Gold cells (Novagen) were transformed with the vector and grown in minimal medium with ${ }^{15} \mathrm{NH}_{4} \mathrm{Cl}$ and $\left[{ }^{13} \mathrm{C}\right]$ glucose as the sole nitrogen and carbon source, respectively. Cells were pelleted, resuspended in PBS, and heat-lysed. Cell debris was removed by centrifugation. The supernatant was dialyzed against Milli-Q water and concentrated. The fusion protein was isolated on a Superdex 75 26/60 column (Amersham Biosciences), equilibrated in $20 \mathrm{mM}$ Tris$\mathrm{HCl}(\mathrm{pH} 8), 0.2 \mathrm{M} \mathrm{NaCl}, 0.01 \%(\mathrm{w} / \mathrm{v}) \mathrm{NaN}_{3}$, concentrated, and digested with Factor Xa (BioLabs) to obtain the peptide. S2 peptide was separated from the GB1 domain and subsequently desalted on a Superdex 30 26/60 column (Amersham Pharmacia Biotech). The yield was $15 \mathrm{mg}$ of S2 peptide from $1 \mathrm{~L}$ minimal medium. Peptide integrity was confirmed by electrospray mass spectrometry.

\section{Sample preparation}

Intact disk membranes were isolated from rod outer segments of bovine retinas using the Ficoll flotation 
method.42 Low ionic strength buffers were used to prevent disk aggregation. Samples were kept on ice and all manipulations were performed in the dark or under dim red light to prevent premature bleaching of rhodop$\mathrm{sin}$. Disks were concentrated to $3.0 \mathrm{mg}$ rhodopsin per $\mathrm{ml}$ in $10 \mathrm{mM}$ Hepes-d18 (Cambridge Isotopes), $20 \mathrm{mM} \mathrm{KCl}$, $50 \mu \mathrm{M}$ DTPA (pH 6.6). Peptide stock solutions at $23 \mathrm{mM}$ were prepared in the same buffer. Appropriate volumes of disk membrane and peptide solutions were combined immediately prior to the NMR experiments. Final sample composition was $2.6 \mathrm{mM}$ S2 peptide and $63 \mu \mathrm{M}$ rhodopsin in $90 \% \mathrm{H}_{2} \mathrm{O}, 10 \%{ }^{2} \mathrm{H}_{2} \mathrm{O}$.

Photo-activation was achieved by subjecting the sample to the intense white light of a focussed microscope light for 60 seconds. Samples were temperatureequilibrated in a water bath for 20 seconds prior to reinsertion into the magnet and data acquisition was started immediately.

\section{NMR spectroscopy}

NMR data were acquired on Bruker spectrometers equipped with a triple resonance three-axis gradient probe. The WATERGATE sequence ${ }^{81}$ was applied for suppression of the water signal. Spectra were processed using NMRPipe software ${ }^{82}$ and Tcl scripts were used for further data analysis.

NOESY spectra were recorded at $750 \mathrm{MHz}{ }^{1} \mathrm{H}$ frequency at $10^{\circ} \mathrm{C}$, using a $48 \mathrm{~ms}$ mixing time. A selective water flip-back pulse was applied during the mixing period. Data matrices with $200 \times 512$ complex points were acquired using acquisition times of $26 \mathrm{~ms}\left(F_{1}\right)$ and $68 \mathrm{~ms}\left(F_{2}\right)$. Quadrature detection in $F_{1}$ was accomplished with the States-TPPI method. ${ }^{83}$ Sine-bell window functions, phase-shifted by $83^{\circ}$ and $72^{\circ}$, were used for processing in the $F_{1}$ and $F_{2}$ dimensions, respectively. Data matrices were zero-filled to $1024 \times 2048$ points.

A 2D NOESY spectrum with 40 transients per free induction decay (FID) was recorded in the presence of dark-adapted rhodopsin, just prior to photo-bleaching. A four-transient NOESY experiment was started immediately after photo-bleaching (total measuring time 38 minutes), using the same sample and identical acquisition parameters. A TrNOESY difference spectrum was calculated by subtracting the scaled NOESY spectrum of the dark-adapted sample from the one recorded on the photo-bleached sample. The scaling factor corrects for the lower diagonal peak intensity in the spectrum recorded after photo-bleaching, caused by strong crossrelaxation in the bound peptide and magnetization transfer from peptide to rhodopsin during the NOE mixing period. However, the optimal scaling factor is not uniform for all interproton vectors of the peptide, and in practice spectra were inspected at multiple scaling factors.

An $r^{-4}$ distance dependence of the NOE cross-peak intensity is assumed in order to minimize the effect of potential spin diffusion on NOE distance restraints. ${ }^{84}$ Intraresidue $\mathrm{H}^{\mathrm{N}}-\mathrm{H}^{\alpha}$ distances of $\sim 2.8 \AA$ are used as reference. Weak cross-peaks between protons $\mathrm{A}$ and $\mathrm{C}$ are ignored if strong cross-peaks between $\mathrm{A}$ and $\mathrm{B}$ and between $\mathrm{B}$ and $\mathrm{C}$ are present. For structure calculation distance constraints of $r \pm 0.2 r$ are used for backbonebackbone and backbone-side-chain interactions, and $r \pm 0.3 r$ for side-chain-side-chain NOEs, respectively. For very weak NOEs, for which $r$ is calculated to be larger than $3.3 \AA$, a more generous tolerance of $\pm 0.06 r^{2} / \AA$ is used. NOE distance errors proportional to $r^{2}$ were previously used by Nilges et al. to minimize the influence of potential spin diffusion on calculated NMR structures. $^{85}$

Proton-coupled ${ }^{1} \mathrm{H}-{ }^{15} \mathrm{~N}$ HSQC spectra were acquired at $600 \mathrm{MHz}{ }^{1} \mathrm{H}$ frequency. The data matrix size was $65 \times 1024$ complex points, resulting in acquisition times of $120 \mathrm{~ms}$ and $114 \mathrm{~ms}$ in $F_{1}$ and $F_{2}$, respectively. Two transients per FID were acquired, giving a measuring time of 9.1 minutes per spectrum. Quadrature in the $F_{1}$ dimension was achieved in the States-TPPI manner. Apodization was performed with $72^{\circ}$-shifted sine-bell $\left(F_{1}\right)$ and squared sine-bell $\left(F_{2}\right)$ window functions. Data matrices were zero-filled to $256 \times 2048$ points.

Proton-coupled ${ }^{1} \mathrm{H}-{ }^{13} \mathrm{C}$ CT-HSQC spectra ${ }^{86,87}$ were recorded at $600 \mathrm{MHz}{ }^{1} \mathrm{H}$ frequency, using a $106 \times 512$ complex points data matrix. Acquisition times of $28 \mathrm{~ms}$ $\left(F_{1}\right)$ and $68 \mathrm{~ms}\left(F_{2}\right)$ were used, with two transients per FID, resulting in a measuring time of 9.3 minutes per spectrum. The inversion pulses during ${ }^{13} \mathrm{C}$ constant time $t_{1}$ evolution were of the adiabatic hyperbolic secant variety $(500 \mu \mathrm{s}$ at $600 \mathrm{MHz})$, with the ${ }^{13} \mathrm{C}$ carrier at $56 \mathrm{ppm}$. Water suppression and $F_{1}$ quadrature were obtained by pulsed field gradient coherence pathway selection. ${ }^{88}$ Data were apodized with $63^{\circ}$-shifted sine-bell $\left(F_{1}\right)$ and squared sine-bell $\left(F_{2}\right)$ windows, prior to zero filling ( $512 \times 2048$ points) and Fourier transformation.

Proton-decoupled ${ }^{31} \mathrm{P}$ NMR spectra of disk membranes $(20 \mathrm{mg}$ disks $/ \mathrm{ml}$ in $10 \mathrm{mM}$ Pipes buffer, $50 \mu \mathrm{M}$ DTPA) were recorded at $242.9 \mathrm{MHz}{ }^{31} \mathrm{P}$ frequency, at $4{ }^{\circ} \mathrm{C}$. 28,000 FIDs were averaged. Proton decoupling was employed with the decoupler frequency tuned to the methylene resonance of the choline headgroup of the lipid.

The ${ }^{31} \mathrm{P}$ NMR resonance frequency of a phospholipid headgroup in a planar membrane fragment, that is characterized by an angle $\alpha$ between the membrane normal and the $B_{0}$ field, is given by $v=v_{\mathrm{i}}+v_{\mathrm{a}}$ $\left(3 \cos ^{2} \alpha-1\right) / 2$, with $v_{\mathrm{a}}=2 / 3\left(v_{\|}-v_{\perp}\right)$, were $\nu_{\mathrm{i}}$ is the isotropic resonance frequency of the lipid while $\nu_{\|}$and $\nu_{\perp}$ are the resonance frequencies observed for parallel and perpendicular mutual orientation of membrane normal and $B_{0}$ field. ${ }^{89}$ Theoretical ${ }^{31} \mathrm{P}$ NMR spectra for oblate spheroidal disk membranes oriented with the short axis parallel with $B_{0}$ were calculated for different aspect ratios of the ellipsoid. Spectra were obtained numerically by integrating the orientation-dependent resonances of small planar membrane fragments over the surface of the ellipsoid. A Gaussian lineshape with a line width parameter $\Delta=\left(v_{\|}-v_{\perp}\right) / 30$ was used. The agreement between measured and calculated spectra was best for an aspect ratio of 2 and $\left(v_{\|}-v_{\perp}\right)=49 \mathrm{ppm}$ (Figure 1). The two extrema in the experimental spectrum are broader than the calculated peaks. For simplicity, a single CSA value of the ${ }^{31} \mathrm{P}$ signal of the lipid was used in the calculation. However, there are three major phospholipid headgroup classes in the disk membrane, i.e. phosphatidylethanolamine $(43( \pm 1) \mathrm{mol} \%)$, phosphatidylcholine $(38( \pm 2) \mathrm{mol} \%)$, and phosphatidylserine $(19( \pm 2) \mathrm{mol} \%)^{90}$ characterized by average ${ }^{31} \mathrm{P}$ CSA values of $38 \mathrm{ppm}, 45 \mathrm{ppm}$, and $54 \mathrm{ppm}$, respectively, at $25^{\circ} \mathrm{C}^{41}$ Slightly larger CSA values are expected at $4{ }^{\circ} \mathrm{C}$, i.e. at lower temperature. ${ }^{91}$

\section{Calculation of peptide structures}

Structure calculations are on the basis of simulated annealing protocols implemented with a modified version of the program X-PLOR, ${ }^{92}$ which allows refinement 
against experimental dipolar couplings $\uparrow^{30,54}$ Pseudopotentials for NOE-derived distances $\left(E_{\mathrm{NOE}}\right)$ and dihedral angles $\left(E_{\text {Dihed }}\right)$ as well as for residual dipolar couplings $\left(E_{\mathrm{DC}}\right)$ are incorporated in the total energy target function $\left(E_{\text {tot }}\right)$ to enforce the experimental restraints:

$$
E_{\mathrm{tot}}=E_{\mathrm{emp}}+E_{\mathrm{NOE}}+E_{\mathrm{Dihed}}+E_{\mathrm{DC}}
$$

where the empirical energy term $E_{\text {emp }}$ describes covalent geometry (bond lengths, bond angles, improper angles) and van der Waals repulsion. No empirical, databasederived energy term was used to increase the quality of the Ramachandran map, ${ }^{93}$ but a database-derived $\chi_{2}=90^{\circ}( \pm 15)^{\circ}$ restraint was used for Phe11. Initial structures were calculated on the basis of NOE-derived restraints only (distances and dihedral angles). The simulated annealing scheme employed closely follows the protocol by Nilges for use with highly ambiguous distance restraints. ${ }^{94}$ Sum-averaging is applied to calculate $E_{\mathrm{NOE}}$, which allows incorporation of highly ambiguous data in early rounds of structure calculations. ${ }^{94}$ Starting coordinates are chosen randomly within a $20 \AA$ cube to avoid any bias. Approximate peptide structures are generated in a NOE-based random search phase. Details on the protocol used are listed in Table 2. Next the obtained structures are regularized using the DGSA protocol listed in the X-PLOR manual. ${ }^{92}$ An NOE-based refinement phase finalizes simulation of the NOE-based peptide structures. A set of 400 NOE-based conformers was generated. The 100 models with the lowest total energy are selected as starting structures for further refinement using both NOE and dipolar coupling restraints (right column in Table 2). Slow cooling is essential at this final stage to reliably localize the global energy minimum that defines the bound peptide conformation.

Peptide structures derived in an initial round of calculations showed several close contacts between protons that were clearly absent in the TrNOESY spectrum. In order to prevent such close contacts, which would be in conflict with the NMR data, eight lower limits of $3.5 \AA$ for these proton-proton distances were used in the final round of calculations.

Five unambiguous $i$ to $i-4$ hydrogen bonds from Leu5-Gly9 to Ile1-Leu5 were clearly recognizable in peptide structures obtained in preliminary structure calculations. In the final round of structure calculations the NOE distance table was supplemented with $\mathrm{HN}^{\mathrm{i}}-\mathrm{O}^{i-4}$ and $\mathrm{N}^{\mathrm{i}}-\mathrm{O}^{i-4}$ distance restraints of $1.9( \pm 0.3) \AA$ and $2.9( \pm 0.4) \AA$, respectively, for these five H-bonds.

In each round of calculations 100 peptide conformations were calculated for a given set of parameters. Subsequently, the 20 structures with lowest total energy were selected for analysis. The computer programs MOLMOL, ${ }^{95}$ PROCHECK-NMR, ${ }^{65}$ and DSSP $^{62}$ were used for visualization and analysis of generated peptide structures. The DSSP program identifies hydrogen bonds on the basis of spatial proximity of potential electron donors and acceptors in the backbone of the simulated structure and uses an energy cut-off of $-0.5 \mathrm{kcal} \mathrm{mol}^{-1}$ to define potential hydrogen bonds.

$\dagger$ Clore, G. M.; Kuszewski, J.; Schwieters, C. D.; Tjandra, N. http://nmr.cit.nih.gov/xplor-nih/

\section{Determination of peptide orientation}

Incorporation of dipolar coupling restraints into the X-PLOR-based simulated annealing protocol follows a previously described procedure. ${ }^{30}$ During structure calculation, peptide coordinates are specified in the laboratory frame. Orientation of the principal axis system of the peptide alignment tensor relative to the laboratory frame is described by means of a rigid, tetraatomic pseudomolecule $O X Y Z$. Bonds $O-X, O-Y$, and $O-Z$ are pairwise orthogonal. The axial component of the alignment tensor is parallel with $O-Z$. Given the tensor components (predefined in the X-PLOR protocol), the orientation of the tensor principal axis system, and the peptide coordinates, the dipolar coupling for any pair of nuclear spins of the generated peptide model can be predicted from equations (3). The dipolar energy penalty term, $E_{\mathrm{DC}}$, accounts for the difference between observed, $D^{\text {obs }}$, and predicted, $D^{\text {calc }}$, dipolar couplings. For backbone amide ${ }^{15} \mathrm{~N}-{ }^{1} \mathrm{H}$ and methine ${ }^{13} \mathrm{C}^{\alpha}-{ }^{1} \mathrm{H}^{\alpha}$ dipolar couplings, $E_{\mathrm{DC}}$ is given by a harmonic potential:

$$
E_{\mathrm{DC}}=k_{\mathrm{DC}}\left(D^{\mathrm{calc}}-D^{\mathrm{obs}}\right)^{2}
$$

Side-chain residual dipolar couplings are treated in a slightly different way in order to account for potential conformational averaging. The magnitude of side-chain couplings may be reduced as a result of a lower order parameter, $\mathbf{S}$, of side-chain relative to backbone bond vectors. For computational simplicity all dipolar couplings of the model are calculated with a uniform $\mathbf{S}$ using equations (3). However, no energy penalty is assigned if the magnitude of $D^{\text {calc }}$ is larger than the magnitude of $D^{\text {obs }}$. This is realized by using a half-open square-well potential for side-chain couplings: ${ }^{49}$

$$
\begin{aligned}
& E_{\mathrm{DC}}=k_{\mathrm{DC}}\left(D^{\text {calc }}-D^{\mathrm{obs}}\right)^{2} \quad \text { for }\left|D^{\text {calc }}\right|<\left|D^{\text {obs }}\right| \\
& E_{\mathrm{DC}}=0 \quad \text { for }\left|D^{\text {calc }}\right| \geq\left|D^{\text {obs }}\right|
\end{aligned}
$$

The orientation of the pseudomolecule OXYZ is allowed to float during simulated annealing with the objective to minimize the target energy function $E_{\text {tot }}$. The force constants for the harmonically restrained dipolar couplings were adjusted to yield root-mean-square deviations in the final structures that are approximately equal to the estimated experimental uncertainty in their measured values.

After completion of the simulated annealing protocol the unique axis, $\mathrm{O}-\mathrm{Z}$, of the axially symmetric alignment tensor of the S2 peptide is parallel with the membrane normal and is used to determine the angle $\beta\left(\beta \leq 90^{\circ}\right)$ between the long axis of the N-terminal helix of the MII-bound S2 peptide and the membrane normal.

\section{Atomic coordinates}

Calculated peptide structures and experimental restraint tables have been deposited with the PDB. The PDB accession code is $1 \mathrm{LVZ}$.

\section{Acknowledgements}

We thank Marius Clore, Klaus Gawrisch, Joachim Granzin, Angela Gronenborn, Stephan Grzesiek, John Kuszewski, Attila Szabo, Nico Tjandra, and Dennis Torchia for stimulating 
discussions and helpful suggestions; Frank Delaglio and Dan Garrett for software support; Sonja Hess for checking peptide purity by mass spectrometry. B.W.K. gratefully acknowledges Georg Büldt, Norbert Dencher, and Stephan Grzesiek for generous support of the project.

\section{References}

1. Hamm, H. E. (1998). The many faces of $G$ protein signaling. J. Biol. Chem. 273, 669-672.

2. Hamm, H. E. (2001). How activated receptors couple to G proteins. Proc. Natl Acad. Sci. USA, 98, 4819-4821.

3. Yeagle, P. L. \& Albert, A. D. (1998). Structure of the G-protein-coupled receptor, rhodopsin: a domain approach. Biochem. Soc. Trans. 26, 520-531.

4. Kisselev, O. G., Meyer, C. K., Heck, M., Ernst, O. P. \& Hofmann, K. P. (1999). Signal transfer from rhodopsin to the G-protein: evidence for a two-site sequential fit mechanism. Proc. Natl Acad. Sci. USA, 96, 4898-4903.

5. Itoh, Y., Cai, K. \& Khorana, H. G. (2001). Mapping of contact sites in complex formation between lightactivated rhodopsin and transducin by covalent crosslinking: use of a chemically preactivated reagent. Proc. Natl Acad. Sci. USA, 98, 4883-4887.

6. Palczewski, K., Kumasaka, T., Hori, T., Behnke, C. A., Motoshima, H., Fox, B. A. et al. (2000). Crystal structure of rhodopsin: a $G$ protein-coupled receptor. Science, 289, 739-745.

7. Lambright, D. G., Sondek, J., Bohm, A., Skiba, N. P., Hamm, H. E. \& Sigler, P. B. (1996). The $2.0 \AA$ crystal structure of a heterotrimeric G protein. Nature, 379, 311-319.

8. Farrens, D. L., Altenbach, C., Yang, K., Hubbell, W. L. \& Khorana, H. G. (1996). Requirement of rigid-body motion of transmembrane helices for light activation of rhodopsin. Science, 274, 768-770.

9. Cai, K., Klein-Seetharaman, J., Farrens, D., Zhang, C., Altenbach, C., Hubbell, W. L. \& Khorana, H. G. (1999). Single-cysteine substitution mutants at amino acid positions 306-321 in rhodopsin, the sequence between the cytoplasmic end of helix VII and the palmitoylation sites: sulfhydryl reactivity and transducin activation reveal a tertiary structure. Biochemistry, 38, 7925-7930.

10. Unger, V. M. \& Schertler, G. F. (1995). Low resolution structure of bovine rhodopsin determined by electron cryo-microscopy. Biophys. J. 68, 1776-1786.

11. Krebs, A., Villa, C., Edwards, P. C. \& Schertler, G. F. (1998). Characterisation of an improved two-dimensional p22 $2_{1}$ crystal from bovine rhodopsin. J. Mol. Biol. 282, 991-1003.

12. Albert, A. D. \& Yeagle, P. L. (2000). Domain approach to three-dimensional structure of rhodopsin using high-resolution nuclear magnetic resonance. Methods Enzymol. 315, 107-115.

13. Loewen, M. C., Klein-Seetharaman, J., Getmanova, E. V., Reeves, P. J., Schwalbe, H. \& Khorana, H. G. (2001). Solution ${ }^{19} \mathrm{~F}$ nuclear Overhauser effects in structural studies of the cytoplasmic domain of mammalian rhodopsin. Proc. Natl Acad. Sci. USA, 98, 4888-4892.

14. Eilers, M., Reeves, P. J., Ying, W., Khorana, H. G. \& Smith, S. O. (1999). Magic angle spinning NMR of the protonated retinylidene Schiff base nitrogen in rhodopsin: Expression of ${ }^{15} \mathrm{~N}$-lysine and ${ }^{13} \mathrm{C}$-glycinelabeled opsin in a stable cell line. Proc. Natl Acad. Sci. USA, 96, 487-492.

15. Grobner, G., Burnett, I. J., Glaubitz, C., Choi, G., Mason, A. J. \& Watts, A. (2000). Observations of light-induced structural changes of retinal within rhodopsin. Nature, 405, 810-813.

16. Hamm, H. E., Deretic, D., Arendt, A., Hargrave, P. A., Koenig, B. \& Hofmann, K. P. (1988). Site of G protein binding to rhodopsin mapped with synthetic peptides from the alpha subunit. Science, 241, 832-835.

17. Kisselev, O. G., Ermolaeva, M. V. \& Gautam, N. (1994). A farnesylated domain in the $G$ protein gamma subunit is a specific determinant of receptor coupling. J. Biol. Chem. 269, 21399-21402.

18. Aris, L., Gilchrist, A., Rens-Domiano, S., Meyer, C., Schatz, P. J., Dratz, E. A. \& Hamm, H. E. (2000). Structural requirements for the stabilization of metarhodopsin II by the carboxyl terminus of the alpha subunit of transducin. J. Biol. Chem. 276, 2333-2339.

19. Dratz, E. A., Furstenau, J. E., Lambert, C. G., Thireault, D. L., Rarick, H., Schepers, T. et al. (1993). NMR structure of a receptor-bound G-protein peptide. Nature, 363, 276-281.

20. Kisselev, O. G., Kao, J., Ponder, J. W., Fann, Y. C., Gautam, N. \& Marshall, G. R. (1998). Light-activated rhodopsin induces structural binding motif in $G$ protein alpha subunit. Proc. Natl Acad. Sci. USA, 95, $4270-4275$.

21. Clore, G. M. \& Gronenborn, A. M. (1998). NMR structure determination of proteins and protein complexes larger than $20 \mathrm{kDa}$. Curr. Opin. Chem. Biol. 2, 564-570.

22. Anglister, J. \& Naider, F. (1991). Nuclear magnetic resonance for studying peptide-antibody complexes by transferred nuclear Overhauser effect difference spectroscopy. Methods Enzymol. 203, 228-241.

23. Zvi, A., Kustanovich, I., Feigelson, D., Levy, R., Eisenstein, M., Matsushita, S. et al. (1995). NMR mapping of the antigenic determinant recognized by an Anti-Gp120, human-immunodeficiency-virus neutralizing antibody. Eur. J. Biochem. 229, 178-187.

24. Gardner, K. H. \& Kay, L. E. (1998). The use of ${ }^{2} \mathrm{H},{ }^{13} \mathrm{C}$ ${ }^{15} \mathrm{~N}$ multidimensional NMR to study the structure and dynamics of proteins. Annu. Rev. Biophys. Biomol. Struct. 27, 357-406.

25. Balaram, P., Bothner-By, A. A. \& Breslow, E. (1973). Nuclear magnetic resonance studies of the interaction of peptides and hormones with bovine neurophysin. Biochemistry, 12, 4695-4704.

26. Clore, G. M. \& Gronenborn, A. M. (1983). Theory of the time dependent transferred nuclear Overhauser effect: Applications to structural analysis of ligandprotein complexes in solution. J. Magn. Reson. 53, 423-442.

27. Campbell, A. P. \& Sykes, B. D. (1993). The twodimensional transferred nuclear Overhauser effect: Theory and practice. Annu. Rev. Biophys. Biomol. Struct. 22, 99-122.

28. Ni, F. \& Zhu, Y. (1994). Accounting for ligand-protein interactions in the relaxation-matrix analysis of transferred nuclear Overhauser effects. J. Magn. Reson. ser. B, 103, 180-184.

29. Zhang, S., Meier, B. H. \& Ernst, R. R. (1992). Polarization echoes in NMR. Phys. Rev. Lett. 69, 2149-2151.

30. Tjandra, N., Omichinski, J. G., Gronenborn, A. M., Clore, G. M. \& Bax, A. (1997). Use of dipolar 
$\mathrm{H}^{1}-\mathrm{N}^{15}$ and $\mathrm{H}^{1}-\mathrm{C}^{13}$ couplings in the structure determination of magnetically oriented macromolecules in solution. Nature Struct. Biol. 4, 732-738.

31. Fischer, M. W. F., Losonczi, J. A., Weaver, J. L. \& Prestegard, J. H. (1999). Domain orientation and dynamics in multidomain proteins from residual dipolar couplings. Biochemistry, 38, 9013-9022.

32. Bewley, C. A. \& Clore, G. M. (2000). Determination of the relative orientation of the two halves of the domain-swapped dimer of cyanovirin- $\mathrm{N}$ in solution using dipolar couplings and rigid body minimization. J. Am. Chem. Soc. 122, 6009-6016.

33. Goto, N. K., Skrynnikov, N. R., Dahlquist, F. W. \& Kay, L. E. (2001). What is the average conformation of bacteriophage T4 lysozyme in solution? A domain orientation study using dipolar couplings measured by solution NMR. J. Mol. Biol. 308, 745-764.

34. Bax, A. \& Tjandra, N. (1997). High-resolution heteronuclear NMR of human ubiquitin in an aqueous liquid crystalline medium. J. Biomol. NMR, 10, 289-292.

35. Clore, G. M., Starich, M. R. \& Gronenborn, A. M. (1998). Measurement of residual dipolar couplings of macromolecules aligned in the nematic phase of a colloidal suspension of rod-shaped viruses. J. Am. Chem. Soc. 120, 10571-10572.

36. Hansen, M. R., Rance, M. \& Pardi, A. (1998). Observation of long-range ${ }^{1} \mathrm{H}-{ }^{1} \mathrm{H}$ distances in solution by dipolar coupling interactions. J. Am. Chem. Soc. 120, 11210-11211.

37. Barrientos, L. G., Louis, J. M. \& Gronenborn, A. M. (2001). Characterization of the cholesteric phase of filamentous bacteriophage fd for molecular alignment. J. Magn. Reson. 149, 154-158.

38. Koenig, B. W., Hu, J. S., Ottiger, M., Bose, S., Hendler, R. W. \& Bax, A. (1999). NMR measurement of dipolar couplings in proteins aligned by transient binding to purple membrane fragments. J. Am. Chem. Soc. 121, 1385-1386.

39. Sass, J., Cordier, F., Hoffmann, A., Cousin, A., Omichinski, J. G., Lowen, H. \& Grzesiek, S. (1999). Purple membrane induced alignment of biological macromolecules in the magnetic field. J. Am. Chem. Soc. 121, 2047-2055.

40. Hong, F. T., Mauzerall, D. \& Mauro, A. (1971). Magnetic anisotropy and the orientation of retinal rods in a homogeneous magnetic field. Proc. Natl Acad. Sci. USA, 68, 1283-1285.

41. Mollevanger, L. C., Dratz, E. A., De Kruijff, B., Hilbers, C. W. \& de Grip, W. J. (1986). 31 ${ }^{\mathrm{P}}$-NMR investigation of magnetically oriented rod outer segments. Spectral analysis and identification of individual phospholipids. Eur. J. Biochem. 156, 383-390.

42. Smith, H. G. \& Litman, B. J. (1982). Preparation of osmotically intact rod outer segment disks by ficoll flotation. Methods Enzymol. 81, 57-61.

43. Koenig, B. W., Mitchell, D. C., Konig, S., Grzesiek, S., Litman, B. J. \& Bax, A. (2000). Measurement of dipolar couplings in a transducin peptide fragment weakly bound to oriented photo-activated rhodopsin. J. Biomol. NMR, 16, 121-125.

44. Arnis, S. \& Hofmann, K. P. (1993). Two Different forms of metarhodopsin II: Schiff-base deprotonation precedes proton uptake and signaling state. Proc. Natl Acad. Sci. USA, 90, 7849-7853.

45. Dickopf, S., Mielke, T. \& Heyn, M. P. (1998). Kinetics of the light-induced proton translocation associated with the $\mathrm{pH}$-dependent formation of the metarho- dopsin I/II equilibrium of bovine rhodopsin. Biochemistry, 37, 16888-16897.

46. Goldman, M. (1984). Interference effects in the relaxation of a pair of unlike spin $-1 / 2$ nuclei. J. Magn. Reson. 60, 437-452.

47. Cone, R. A. (1972). Rotational diffusion of rhodopsin in the visual receptor membrane. Nature New Biol. 236, 39-43.

48. Cavanagh, J., Fairbrother, W. J., Palmer, A. G. \& Skelton, N. J. (1996). Protein NMR Spectroscopy: Principles and Practice, Academic Press, San Diego.

49. Ottiger, M., Delaglio, F., Marquardt, J. L., Tjandra, N. \& Bax, A. (1998). Measurement of dipolar couplings for methylene and methyl sites in weakly oriented macromolecules and their use in structure determination. J. Magn. Reson. 134, 365-369.

50. Lipari, G. \& Szabo, A. (1982). Model-free approach to the interpretation of nuclear magnetic resonance relaxation in macromolecules. 1. Theory and range of validity. J. Am. Chem. Soc. 104, 4546-4559.

51. Tjandra, N. \& Bax, A. (1997). Direct measurement of distances and angles in biomolecules by NMR in a dilute liquid crystalline medium. Science, 278, $1111-1114$.

52. Bax, A., Kontaxis, G. \& Tjandra, N. (2001). Dipolar couplings in macromolecular structure determination. Methods Enzymol. 339, 127-174.

53. Clore, G. M., Gronenborn, A. M. \& Bax, A. (1998). A robust method for determining the magnitude of the fully asymmetric alignment tensor of oriented macromolecules in the absence of structural information. J. Magn. Reson. 133, 216-221.

54. Clore, G. M., Gronenborn, A. M. \& Tjandra, N. (1998). Direct structure refinement against residual dipolar couplings in the presence of rhombicity of unknown magnitude. J. Magn. Reson. 131, 159-162.

55. Zweckstetter, M. \& Bax, A. (2002). Evaluation of uncertainty in alignment tensors obtained from dipolar couplings. J. Biomol. NMR, 23, 127-137.

56. Driscoll, P. C., Clore, G. M., Marion, D., Wingfield, P. T. \& Gronenborn, A. M. (1990). Complete resonance assignment for the polypeptide backbone of interleukin-1-beta using 3-dimensional heteronuclear NMR-spectroscopy. Biochemistry, 29, 3542-3556.

57. Anglister, J., Levy, R. \& Scherf, T. (1989). Interactions of antibody aromatic residues with a peptide of cholera toxin observed by two-dimensional transferred nuclear Overhauser effect difference spectroscopy. Biochemistry, 28, 3360-3365.

58. Senn, H., Werner, B., Messerle, B. A., Weber, C., Traber, R., Wüthrich, K. \& Senn, H. (1989). Stereospecific assignments of the methyl ${ }^{1} \mathrm{H}$ NMR lines of valine and leucine in polypeptides by nonrandom ${ }^{13} \mathrm{C}$ labeling. FEBS Letters, 249, 113-118.

59. Zhang, O. W. \& FormanKay, J. D. (1997). NMR studies of unfolded states of an SH3 domain in aqueous solution and denaturing conditions. Biochemistry, 36, 3959-3970.

60. Dunbrack, R. L. \& Karplus, M. (1994). Conformationalanalysis of the backbone-dependent rotamer preferences of protein side-chains. Nature Struct. Biol. 1, 334-340.

61. Gagne, S. M., Tsuda, S., Li, M. X., Chandra, M., Smillie, L. B. \& Sykes, B. D. (1994). Quantification of the calcium-induced secondary structural-changes in the regulatory domain of troponin-C. Protein Sci. 3, 1961-1974.

62. Kabsch, W. \& Sander, C. (1983). Dictionary of protein secondary structure: pattern recognition 
of hydrogen-bonded and geometrical features. Biopolymers, 22, 2577-2637.

63. Aurora, R., Srinivasan, R. \& Rose, G. D. (1994). Rules for $\alpha$-helix termination by glycine. Science, 264, 1126-1130.

64. Schellman, C. (1980). The $\alpha_{\mathrm{L}}$ conformation at the ends of helices. In Protein Folding (Jaenicke, R., ed.), pp. 53-61, Elsevier, Amsterdam.

65. Laskowski, R. A., Rullmann, J. A. C., MacArthur, M. W., Kaptein, R. \& Thornton, J. M. (1996). AQUA and procheck NMR: Programs for checking the quality of protein structures solved by NMR. J. Biomol. NMR, 8, 477-486.

66. Martin, E. L., Rens-Domiano, S., Schatz, P. J. \& Hamm, H. E. (1996). Potent peptide analogues of a $G$ protein receptor-binding region obtained with a combinatorial library. J. Biol. Chem. 271, 361-366.

67. Illing, M., Molday, L. L. \& Molday, R. S. (1997). The $220-\mathrm{kDa}$ rim protein of retinal rod outer segments is a member of the $\mathrm{ABC}$ transporter superfamily. J. Biol. Chem. 272, 10303-10310.

68. Worcester, D. L. (1978). Structural origins of diamagnetic anisotropy in proteins. Proc. Natl Acad. Sci. USA, 75, 5475-5477.

69. Noel, J. P., Hamm, H. E. \& Sigler, P. B. (1993). The $2.2 \AA$ crystal structure of transducin-alpha complexed with GTP gamma S. Nature, 366, 654-663.

70. Lambright, D. G., Noel, J. P., Hamm, H. E. \& Sigler, P. B. (1994). Structural determinants for activation of the alpha-subunit of a heterotrimeric $G$ protein. Nature, 369, 621-628.

71. Arepalli, S. R., Glaudemans, C. P. J., Daves, G. D., Kovac, P. \& Bax, A. (1995). Identification of proteinmediated indirect NOE effects in a disaccharideFab' complex by transferred ROESY. J. Magn. Reson. ser. B, 106, 195-198.

72. Dratz, E. A., Furstenau, J. E., Lambert, C. G., Thireault, D. L., Rarick, H., Schepers, T. et al. (1997). NMR structure of a receptor-bound G-protein peptide (correction). Nature, 390, 424.

73. Richardson, J. S. \& Richardson, D. C. (1988). Amino acid preferences for specific locations at the ends of alpha helices. Science, 240, 1648-1652.

74. Laskowski, R. A., MacArthur, M. W., Moss, D. S. \& Thornton, J. W. (1993). PROCHECK: A program to check the stereochemical quality of protein structures. J. Appl. Crystallog. 26, 283-291.

75. Cai, K., Itoh, Y. \& Khorana, H. G. (2001). Mapping of contact sites in complex formation between transducin and light-activated rhodopsin by covalent crosslinking: Use of a photoactivatable reagent. Proc. Natl Acad. Sci. USA, 98, 4877-4882.

76. Ernst, O. P., Meyer, C. K., Marin, E. P., Henklein, P., Fu, W. Y., Sakmar, T. P. \& Hofmann, K. P. (2000). Mutation of the fourth cytoplasmic loop of rhodopsin affects binding of transducin and peptides derived from the carboxyl-terminal sequences of transducin $\alpha$ and $\gamma$ subunits. J. Biol. Chem. 275, 1937-1943.

77. Yeagle, P. L., Choi, G. \& Albert, A. D. (2001). Studies on the structure of the G-protein-coupled receptor rhodopsin including the putative G-protein binding site in unactivated and activated forms. Biochemistry, 40, 11932-11937.

78. Konig, B., Arendt, A., McDowell, J. H., Kahlert, M., Hargrave, P. A. \& Hofmann, K. P. (1989). Three cytoplasmic loops of rhodopsin interact with transducin. Proc. Natl Acad. Sci. USA, 86, 6878-6882.
79. Franke, R. R., Sakmar, T. P., Graham, R. M. \& Khorana, H. G. (1992). Structure and function in rhodopsin. Studies of the interaction between the rhodopsin cytoplasmic domain and transducin. J. Biol. Chem. 267, 14767-14774.

80. Huth, J. R., Bewley, C. A., Jackson, B. M., Hinnebusch, A. G., Clore, G. M. \& Gronenborn, A. M. (1997). Design of an expression system for detecting folded protein domains and mapping macromolecular interactions by NMR. Protein Sci. 6, 2359-2364.

81. Piotto, M., Saudek, V. \& Sklenár, V. (1992). Gradienttailored excitation for single-quantum NMR spectroscopy of aqueous sloutions. J. Biomol. NMR, 2, 661-665.

82. Delaglio, F., Grzesiek, S., Vuister, G. W., Zhu, G., Pfeifer, J. \& Bax, A. (1995). NMRPipe: A multidimensional spectral processing system based on UNIX pipes. J. Biomol. NMR, 6, 277-293.

83. Marion, D., Ikura, M., Tschudin, R. \& Bax, A. (1989). Rapid recording of 2D NMR-spectra without phase cycling-application to the study of hydrogen-exchange in proteins. J. Magn. Reson. 85, 393-399.

84. Guntert, P., Braun, W. \& Wuthrich, K. (1991). Efficient computation of 3-dimensional protein structures in solution from nuclear-magnetic-resonance data using the program DIANA and the supporting programs CALIBA, HABAS and GLOMSA. J. Mol. Biol. 217, 517-530.

85. Nilges, M., Macias, M. J., Odonoghue, S. I. \& Oschkinat, H. (1997). Automated NOESY interpretation with ambiguous distance restraints: the refined NMR solution structure of the pleckstrin homology domain from beta-spectrin. J. Mol. Biol. 269, 408-422.

86. Santoro, J. \& King, G. C. (1992). A constant-time 2D Overbodenhausen experiment for inverse correlation of isotopically enriched species. J. Magn. Reson. 97, 202-207.

87. Vuister, G. W. \& Bax, A. (1992). Resolution enhancement and spectral editing of uniformly ${ }^{13} \mathrm{C}$-enriched proteins by homonuclear broad-band ${ }^{13} \mathrm{C}$ decoupling. J. Magn. Reson. 98, 428-435.

88. Kay, L. E., Keifer, P. \& Saarinen, T. (1992). Pure absorption gradient enhanced heteronuclear single quantum correlation spectroscopy with improved sensitivity. J. Am. Chem. Soc. 114, 10663-10665.

89. Seelig, J. (1978). $31^{\mathrm{P}}$ nuclear magnetic resonance and the head group structure of phospholipids in membranes. Biochim. Biophys. Acta, 515, 105-140.

90. Mollevanger, L. C. P. J. \& De Grip, W. J. (1984). Phase behavior of isolated photoreceptor membrane lipids is modulated by bivalent cations. FEBS Letters, 169, $256-260$

91. Niederberger, W. \& Seelig, J. (1976). Phosphorus-31 chemical shift anisotropy in unsonicated bilayers. J. Am. Chem. Soc. 98, 3704-3706.

92. Brunger, A. T. (1993). XPLOR: A System for X-ray Crystallography and NMR, Yale University Press, New Haven.

93. Kuszewski, J., Gronenborn, A. M. \& Clore, G. M. (1997). Improvements and extensions in the conformational database potential for the refinement of NMR and X-ray structures of proteins and nucleic acids. J. Magn. Reson. 125, 171-177.

94. Nilges, M. (1995). Calculation of protein structures with ambiguous distance restraints. Automated 
assignment of ambiguous NOE crosspeaks and disulphide connectivities. J. Mol. Biol. 245, 645-660.

95. Koradi, R., Billeter, M. \& Wuthrich, K. (1996).

MOLMOL: A program for display and analysis of

macromolecular structures. J. Mol. Graph. 14, 51-55.
96. Berendsen, H. J. C., Postma, J. P. M., van Gunsteren, W. F., DiNola, A. \& Haak, J. R. (1984). Molecular dynamics with coupling to an external bath. J. Chem. Phys. 81, 3684-3690.

Edited by P. Wright

(Received 26 April 2002; received in revised form 11 July 2002; accepted 15 July 2002) 